\title{
Norm-constrained Unscented Kalman Filter with Application to High Area-to-Mass Ratio Space-Debris Tracking
}

\author{
Stephen Alexander Chee* and James Richard Forbes ${ }^{\dagger}$ \\ McGill University, Montreal, QC, H3A 0C3, Canada
}

\begin{abstract}
In this paper a norm-constrained unscented Kalman filter that enforces a state normconstraint is presented. This filter is an extension of the norm-constrained Kalman filter presented by Zanetti et al. The proposed norm-constrained Kalman Filter is used to estimate the pose of a High Area-to-Mass Ratio (HAMR) space-debris object. In much of the literature, the pose and other properties of space-debris objects are estimated assuming that the debris object being observed is a cuboid or some other convex multifaceted object. However, the literature also makes the case that a portion of the HAMR objects found near the Geosynchronous orbit (GEO) belt are fragments of multi-layered insulation (MLI) blankets that have separated from their parent satellites. These objects are slender and are appropriately modeled as thin plates. In this paper, simulated estimation results are presented demonstrating that the tumbling motion of these plate-like objects cannot be reasonably captured by light curve observations from a single observation site. However, by combining together measurements from multiple observation sites, estimation of these tumbling motions is possible. This observation further motivates the development of projects like DARPA's OrbitOutlook program to add more diverse locations for sensors and implement a central database to collect this data.
\end{abstract}

\section{Introduction}

With events such as the collision of a Cosmos satellite and an Iridium satellite in 2009, and the break up of the Fengyun 1C satellite in 2007, there has been a renewed interest in improving the space debris catalog in order to avoid collisions with operational space assets. ${ }^{1}$ Through observational surveys, a particular class of High Area-to-Mass Ratio (HAMR) objects near the Geosynchronous Earth orbit (GEO) belt and in Geosynchronous transfer orbits (GTO) have been found and are of concern. ${ }^{2}$ These space objects (SOs) are in high Earth orbits and as such the effect of atmospheric drag is insufficient to naturally de-orbit these objects, making them a continual collision threat to assets in the GEO belt. Furthermore, the high areato-mass ratio of these objects make their orbits subject to large perturbations due to the effects of solar radiation pressure (SRP).

Propagating estimates of the orbital trajectory of these SOs within reasonable limits of uncertainty is necessary if infrequent observations over long periods of time are to be correlated and the SOs are to be cataloged. ${ }^{1}$ A complicating factor in the propagation of estimates of the states of these SOs is the coupling of the attitude and orbital dynamics through the SRP. The coupled attitude and orbital dynamics of these debris makes estimating the attitude of these objects desirable in order to improve the propagation of estimates of these objects' orbital trajectory. Unfortunately, objects in high-altitude orbits are too small and faint for optical sensors to provide high enough resolutions to infer their attitude based on feature detection. However, the temporal brightness of these objects can be readily measured. Since these objects are not self illuminating, but rather reflect light from the Sun, their apparent brightness will be dependent on their shape and attitude allowing for these characteristics to be inferred through observation of their brightness. ${ }^{3}$ These measurements of changes in brightness over time are called light curves.

*Ph.D. Candidate. Department of Mechanical Engineering, 817 Sherbrooke Street West, Macdonald Engineering Building, Montreal QC. H3A 0C3, Email: stephen.chee@mail.mcgill.ca

${ }^{\dagger}$ Assistant Professor. Department of Mechanical Engineering, Email: james.richard.forbes@mcgill.ca 
To use light curves to estimate the attitude of these space-debris objects, a measurement model is required to compare the propagated estimate of the state of the space object to an actual measurement. Since these objects are reflecting light from the Sun, their brightness can be modeled using bidirectional reflectance distribution functions (BRDFs). ${ }^{4,5}$ In Ref. 4, it was shown that light curves can be used to infer the attitude and reflectance properties of a SO via an unscented Kalman filter (UKF). In Ref. 5, it was demonstrated that combining light curve and angles measurements of the SO (its azimuth and elevation relative to the observation site) allowed for the object's mass and albedo areas to be estimated using a UKF. In Ref. 3, a Multiple-Model estimation framework was implemented to determine the attitude and position of a SO with uncertain knowledge of the SO's shape. Holzinger et al. ${ }^{6}$ present a particle filter to track actively maneuvering satellites with shape uncertainty using light curves by incorporating uncertainty in angular velocities as process noise to account for maneuvering dynamics. Efforts have been made to estimate optical parameters of SOs as well as SOs' attitudes from real measurements. For example, in Ref. 7, measurements from the AMOS telescope in Hawaii were used to estimate the surface albedo and specular reflectance of spherical satellites and droplets of sodium potassium alloy in orbit. In Ref. 8, the angular speed and axis of rotation of two disposed upper stages of rockets of cylindrical shape were estimated using real optical measurements.

It is believed that the source of some of the uncorrelated HAMR objects near the GEO belt is space debris in the form of damaged satellites and multilayer insulation (MLI) blankets broken off from satellites in GEO. ${ }^{2}$ Currently, efforts to propagate these observed objects between measurements over long time spans so that they can be cataloged have had limited success. ${ }^{9}, 10$ To this end, work has been done to develop higher-fidelity dynamics models that capture the motions of MLI debris. In Ref. 10, the attitude of HAMR objects assumed to be flat rigid plates were simulated in the presence of SRP and third-body gravitational perturbations. Using this model, simulated light curve measurements were made and compared to actual observations made with an optical telescope. Further work has been done to incorporate the effects of Earth shadowing ${ }^{11}$ and self shadowing. ${ }^{12}$ In Ref. 13, a semi-coupled approach to propagating the attitude and orbital trajectory of a flat plate in a high altitude orbit is outlined yielding computational savings when compared to propagating the fully coupled dynamics for long time scales.

If these HAMR space-debris objects are best modeled as plates rather than fully three-dimensional objects, then estimating their attitude using light curves will become more difficult since a plate only has two surfaces reflecting light resulting in a less rich light curve dataset than would be seen from a multifaceted three-dimensional object as studied in the literature. ${ }^{3,4,6,14}$ As a result, the convergence of a state estimator such as an unscented Kalman filter (UKF), will require initial conditions relatively close to the true state. However, in this paper, it is shown that a larger error in the initial guess of the UKF can still lead to convergence to the true state if concurrent measurements from multiple observation sites are combined. The current state of the Space Surveillance Network makes this data gathering scenario difficult, however with the development of programs such as DARPA's OrbitOutlook, ${ }^{15}$ a network with more diverse locations for sensors and a central database for the collection of data can enable greater success in the tracking of these HAMR objects. A previous study by Piergentili et al. ${ }^{16}$ demonstrating improved orbit determination through estimating the object's position and velocity using angles measurements from two observation sites further supports the advantages of a diverse network of sensors for tracking space debris.

Estimating the pose of a space object involves estimating both its position and its attitude. When estimating attitude, the use of a four-parameter quaternion is often employed instead of a three-term parameterization, since three-term parameterizations suffer from singularities in the relationship between their time-rate-of-change and angular velocity. Using a quaternion complicates the application of techniques derived from the Kalman filter if it is desired to enforce the quaternion norm constraint. One way to account for the quaternion norm constraint is to use a multiplicative scheme in which local small changes in attitude are calculated using a three-term parameterization that is then used to update the global attitude estimate through quaternion multiplication. ${ }^{17,18}$ Alternatively, the quaternion can be estimated directly using methods that modify the Kalman filter framework to enforce the state constraint. Two popular approaches for addressing equality state constraints within the Kalman filter framework are the pseudomeasurement method and the projection method. The pseudomeasurement method modifies the Kalman filter by considering the constraint to be a perfect noiseless measurement. ${ }^{19-21}$ This modification however can cause the measurement noise covariance to be singular and lead to numerical problems during implementation. ${ }^{22}$ The projection method presented in Ref. 23 enforces linear equality constraints by projecting the unconstrained solution to the Kalman filter onto a constraint surface. This work was extended to accommodate nonlinear 
equality constraints in Ref. 24. In Ref. 25, the projection method is applied to the UKF. Further information concerning these methods can be found in Ref. 22. In this paper the method presented by Zanetti et al. in Ref. 26 is modified to be used in the UKF. This method modifies the Kalman gain matrix using a Lagrange multiplier to enforce the norm-constraint as a constrained optimization problem in the correction step.

This paper is organized as follows. In Section II, the Kalman filter and unscented Kalman filter are reviewed. In Section III, the method for deriving the norm-constrained EKF presented in Ref. 26 is outlined and modified for use in the UKF. Finally, in Section IV the dynamics and measurement models for the space-debris tracking problem are described and simulation and state estimation results are presented.

\section{Preliminaries: Kalman Filter and Unscented Kalman Filter}

Consider the linear system

$$
\begin{aligned}
\mathbf{x}_{k} & =\mathbf{F}_{k-1} \mathbf{x}_{k-1}+\boldsymbol{\Gamma}_{w, k-1} \mathbf{w}_{k-1}, \\
\mathbf{y}_{k} & =\mathbf{H}_{k} \mathbf{x}_{k}+\boldsymbol{\Gamma}_{n, k} \mathbf{n}_{k},
\end{aligned}
$$

where $\mathbf{x}_{k}$ is the system's state at time $k, \mathbf{w}_{k}$ is the Gaussian process noise with zero mean and covariance $\mathbf{Q}_{k}, \mathbf{y}_{k}$ is the measurement at time $k$, and $\mathbf{n}_{k}$ is the Gaussian measurement noise with zero mean and covariance $\mathbf{R}_{k}$. The Kalman filter applied to this system has a prediction-correction structure (Ref. 27, p. 318-320):

Prediction:

$$
\begin{aligned}
\hat{\mathbf{x}}_{k \mid k-1} & =\mathbf{F}_{k-1} \hat{\mathbf{x}}_{k-1 \mid k-1}, \\
\mathbf{P}_{k \mid k-1}^{x x} & =\mathcal{E}\left\{\left(\mathbf{x}_{k}-\hat{\mathbf{x}}_{k \mid k-1}\right)\left(\mathbf{x}_{k}-\hat{\mathbf{x}}_{k \mid k-1}\right)^{\top}\right\} \\
& =\mathbf{F}_{k-1} \mathbf{P}_{k-1 \mid k-1}^{x x} \mathbf{F}_{k-1}^{\top}+\boldsymbol{\Gamma}_{w, k-1} \mathbf{Q}_{k-1} \boldsymbol{\Gamma}_{w, k-1}^{\top},
\end{aligned}
$$

Correction:

$$
\begin{aligned}
\hat{\mathbf{y}}_{k \mid k-1} & =\mathbf{H}_{k} \hat{\mathbf{x}}_{k \mid k-1}, \\
\mathbf{P}_{k \mid k-1}^{x y} & =\mathcal{E}\left\{\left(\mathbf{x}_{k}-\hat{\mathbf{x}}_{k \mid k-1}\right)\left(\mathbf{y}_{k}-\hat{\mathbf{y}}_{k \mid k-1}\right)^{\top}\right\} \\
& =\mathbf{P}_{k \mid k-1}^{x x} \mathbf{H}_{k}^{\top}, \\
\mathbf{P}_{k \mid k-1}^{y y} & =\mathcal{E}\left\{\left(\mathbf{y}_{k}-\hat{\mathbf{y}}_{k \mid k-1}\right)\left(\mathbf{y}_{k}-\hat{\mathbf{y}}_{k \mid k-1}\right)^{\top}\right\} \\
& =\mathbf{H}_{k} \mathbf{P}_{k \mid k-1}^{x x} \mathbf{H}_{k}^{\top}+\boldsymbol{\Gamma}_{n, k} \mathbf{R}_{k} \boldsymbol{\Gamma}_{n, k}^{\top}, \\
\mathbf{K}_{k} & =\mathbf{P}_{k \mid k-1}^{x y}\left(\mathbf{P}_{k \mid k-1}^{y y}\right)^{-1}, \\
\hat{\mathbf{x}}_{k \mid k} & =\hat{\mathbf{x}}_{k \mid k-1}+\mathbf{K}_{k}\left(\mathbf{y}_{k}-\mathbf{H}_{k} \hat{\mathbf{x}}_{k \mid k-1}\right), \\
\mathbf{P}_{k \mid k}^{x x} & =\mathcal{E}\left\{\left(\mathbf{x}_{k}-\hat{\mathbf{x}}_{k \mid k}\right)\left(\mathbf{x}_{k}-\hat{\mathbf{x}}_{k \mid k}\right)^{\top}\right\} \\
& =\mathbf{P}_{k \mid k-1}^{x x}-\mathbf{P}_{k \mid k-1}^{x y}\left(\mathbf{P}_{k \mid k-1}^{y y}\right)^{-1}\left(\mathbf{P}_{k \mid k-1}^{x y}\right)^{\top} \\
& =\mathbf{P}_{k \mid k-1}^{x x}-\mathbf{K}_{k} \mathbf{P}_{k \mid k-1}^{y y} \mathbf{K}_{k}^{\top},
\end{aligned}
$$

where $\mathcal{E}\{\cdot\}$ denotes the expectation. The Kalman filter is optimal in that the Kalman gain matrix $\mathbf{K}_{k}$ minimizes the objective function $J_{k}$ where

$$
J_{k}=\operatorname{trace}\left(\mathbf{P}_{k \mid k}^{x x}\right) .
$$

To simplify notation, let $\hat{\mathbf{x}}_{k}=\hat{\mathbf{x}}_{k \mid k}, \hat{\mathbf{x}}_{k}^{-}=\hat{\mathbf{x}}_{k \mid k-1}, \hat{\mathbf{y}}_{k}^{-}=\hat{\mathbf{y}}_{k \mid k-1}, \mathbf{P}_{k}^{-}=\mathbf{P}_{k \mid k-1}^{x x}$, and $\mathbf{P}_{k}=\mathbf{P}_{k \mid k}^{x x}$.

Consider the the nonlinear system

$$
\begin{aligned}
& \mathbf{x}_{k}=\mathbf{f}_{k-1}\left(\mathbf{x}_{k-1}, \mathbf{w}_{k-1}\right), \\
& \mathbf{y}_{k}=\mathbf{h}_{k}\left(\mathbf{x}_{k}, \mathbf{n}_{k}\right) .
\end{aligned}
$$

The Kalman filter can be modified in one of several ways in order to estimate the states of this nonlinear system. Two popular methods include linearizing the dynamics and measurement equations, arriving at the Extended Kalman Filter (EKF), or the statistics can be approximated using the unscented transformation arriving at the unscented Kalman filter (UKF) (Ref. 27, p. 433). 
In the prediction and correction steps, the unscented Kalman filter generates a set of sigma points $\mathcal{Z}_{i}$ using the Cholesky decomposition and combines these sigma points to calculate the means and covariances (Ref. 28, p. 89). The UKF also has a prediction-correction structure:

Prediction:

$$
\begin{aligned}
\mathbf{z} & =\left[\begin{array}{c}
\hat{\mathbf{x}}_{k-1} \\
\mathbf{0}
\end{array}\right], \quad \mathbf{Y}=\left[\begin{array}{cc}
\mathbf{P}_{k-1} & \mathbf{0} \\
\mathbf{0} & \mathbf{Q}_{k-1}
\end{array}\right], \\
\mathbf{S S}^{\boldsymbol{\top}} & =\mathbf{Y} \\
\mathcal{Z}_{0} & =\mathbf{z} \\
\mathcal{Z}_{i} & =\mathbf{z}+\sqrt{L+\kappa} \operatorname{col}_{i} \mathbf{S} \quad i=1, \ldots, L, \\
\mathcal{Z}_{i+L} & =\mathbf{z}-\sqrt{L+\kappa} \operatorname{col}_{i} \mathbf{S}, \quad i=0, \ldots, 2 L, \\
\mathcal{Z}_{i} & =\left[\begin{array}{c}
\mathcal{X}_{i, k-1} \\
\mathcal{W}_{i, k-1}
\end{array}\right], \quad \mathcal{X}_{i, k}^{-}=\mathbf{f}_{k-1}\left(\mathcal{X}_{i, k-1}, \mathcal{W}_{i, k-1}\right) \quad i=0, \\
\hat{\mathbf{x}}_{k}^{-} & =\frac{1}{L+\kappa}\left(\kappa \mathcal{X}_{0, k}^{-}+\frac{1}{2} \sum_{i=1}^{2 L} \mathcal{X}_{i, k}^{-}\right), \\
\mathbf{P}_{k}^{-} & =\frac{1}{L+\kappa}\left(\kappa\left(\mathcal{X}_{0, k}^{-}-\hat{\mathbf{x}}_{k}^{-}\right)\left(\mathcal{X}_{0, k}^{-}-\hat{\mathbf{x}}_{k}^{-}\right)^{\top}+\frac{1}{2} \sum_{i=1}^{2 L}\left(\mathcal{X}_{i, k}^{-}-\hat{\mathbf{x}}_{k}^{-}\right)\left(\mathcal{X}_{i, k}^{-}-\hat{\mathbf{x}}_{k}^{-}\right)^{\top}\right),
\end{aligned}
$$

Correction:

$$
\begin{aligned}
\mathbf{z} & =\left[\begin{array}{c}
\hat{\mathbf{x}}_{k}^{-} \\
\mathbf{0}
\end{array}\right], \quad \mathbf{Y}=\left[\begin{array}{cc}
\mathbf{P}_{k}^{-} & \mathbf{0} \\
\mathbf{0} & \mathbf{R}_{k}
\end{array}\right], \\
\mathbf{S S}^{\top} & =\mathbf{Y} \\
\mathcal{Z}_{0} & =\mathbf{z} \\
\mathcal{Z}_{i} & =\mathbf{z}+\sqrt{L+\kappa} \operatorname{col}_{i} \mathbf{S} \quad i=1, \ldots, L \\
\mathcal{Z}_{i+L} & =\mathbf{z}-\sqrt{L+\kappa} \operatorname{col}_{i} \mathbf{S}, \\
\mathcal{Z}_{i} & =\left[\begin{array}{l}
\mathcal{X}_{i, k}^{-} \\
\mathcal{N}_{i, k}
\end{array}\right], \quad \mathcal{Y}_{i, k}^{-}=\mathbf{h}_{k}\left(\mathcal{X}_{i, k}^{-}, \mathcal{N}_{i, k}\right) \\
\hat{\mathbf{y}}_{k}^{-} & =\frac{1}{L+\kappa}\left(\kappa \mathcal{Y}_{0, k}^{-}+\frac{1}{2} \sum_{i=1}^{2 L} \mathcal{Y}_{i, k}^{-}\right) \\
\mathbf{U}_{k} & =\frac{1}{L+\kappa}\left(\kappa\left(\mathcal{X}_{0, k}^{-}-\hat{\mathbf{x}}_{k}^{-}\right)\left(\mathcal{Y}_{0, k}^{-}-\hat{\mathbf{y}}_{k}^{-}\right)^{\top}+\frac{1}{2} \sum_{i=1}^{2 L}\left(\mathcal{X}_{i, k}^{-}-\hat{\mathbf{x}}_{k}^{-}\right)\left(\mathcal{Y}_{i, k}^{-}-\hat{\mathbf{y}}_{k}^{-}\right)^{\top}\right) \\
\mathbf{V}_{k} & =\frac{1}{L+\kappa}\left(\kappa\left(\mathcal{Y}_{0, k}^{-}-\hat{\mathbf{y}}_{k}^{-}\right)\left(\mathcal{Y}_{0, k}^{-}-\hat{\mathbf{y}}_{k}^{-}\right)^{\top}+\frac{1}{2} \sum_{i=1}^{2 L}\left(\mathcal{Y}_{i, k}^{-}-\hat{\mathbf{y}}_{k}^{-}\right)\left(\mathcal{Y}_{i, k}^{-}-\hat{\mathbf{y}}_{k}^{-}\right)^{\top}\right) \\
\mathbf{P}_{k \mid k-1}^{y y} & \approx \mathbf{V}_{k}, \\
\mathbf{P}_{k \mid k-1}^{x y} & \approx \mathbf{U}_{k}, \\
\mathbf{K}_{k} & =\mathbf{U}_{k} \mathbf{V}_{k}^{-1}
\end{aligned}
$$




$$
\begin{aligned}
\hat{\mathbf{x}}_{k} & =\hat{\mathbf{x}}_{k}^{-}+\mathbf{K}_{k}\left(\mathbf{y}_{k}-\hat{\mathbf{y}}_{k}^{-}\right), \\
\mathbf{P}_{k} & =\mathbf{P}_{k}^{-}-\mathbf{K}_{k} \mathbf{V}_{k} \mathbf{K}_{k}^{\top} \\
& =\mathbf{P}_{k}^{-}-\mathbf{K}_{k} \mathbf{U}_{k}^{\top}-\mathbf{U}_{k} \mathbf{K}_{k}^{\top}+\mathbf{K}_{k} \mathbf{V}_{k} \mathbf{K}_{k}^{\top} .
\end{aligned}
$$

\section{Norm-constrained UKF}

Consider the case where the system's state satisfies the norm-constraint

$$
\mathbf{x}_{k}^{\top} \mathbf{x}_{k}-\ell^{2}=0 .
$$

It is then desired that the posterior state estimate also satisfy the constraint

$$
\begin{gathered}
\hat{\mathbf{x}}_{k}^{\top} \hat{\mathbf{x}}_{k}-\ell^{2}=0, \\
\left(\hat{\mathbf{x}}_{k}^{-}+\mathbf{K}_{k} \boldsymbol{\rho}_{k}\right)^{\top}\left(\hat{\mathbf{x}}_{k}^{-}+\mathbf{K}_{k} \boldsymbol{\rho}_{k}\right)-\ell^{2}=0, \\
\left(\hat{\mathbf{x}}_{k}^{-}\right)^{\top} \hat{\mathbf{x}}_{k}^{-}+2\left(\hat{\mathbf{x}}_{k}^{-}\right)^{\top} \mathbf{K}_{k} \boldsymbol{\rho}_{k}+\boldsymbol{\rho}_{k}^{\top} \mathbf{K}_{k}^{\top} \mathbf{K}_{k} \boldsymbol{\rho}_{k}-\ell^{2}=0,
\end{gathered}
$$

where $\boldsymbol{\rho}_{k}=\mathbf{y}_{k}-\mathbf{h}_{k}\left(\hat{\mathbf{x}}_{k}^{-}, \mathbf{0}\right)$ is the innovation. To enforce this constraint, the same approach taken by Zanetti et al. in Ref. 26 will be taken, where the objective function in Eq. (1) is augmented with a Lagrange multiplier resulting in the optimization problem

$$
\hat{J}_{k}^{*}=\min _{\mathbf{K}_{k}}\left\{\hat{J}_{k}\right\} \quad \text { where } \quad \hat{J}_{k}=\operatorname{trace}\left(\mathbf{P}_{k}\right)+\lambda_{k}\left(\hat{\mathbf{x}}_{k}^{\top} \hat{\mathbf{x}}_{k}-\ell^{2}\right) .
$$

Expressing $\mathbf{P}_{k}$ in terms of $\mathbf{K}_{k}$ yields

$$
\begin{aligned}
\mathbf{P}_{k} & =\mathcal{E}\left[\left(\mathbf{x}_{k}-\hat{\mathbf{x}}_{k}\right)\left(\mathbf{x}_{k}-\hat{\mathbf{x}}_{k}\right)^{\top}\right] \\
& =\mathcal{E}\left\{\left[\left(\mathbf{x}_{k}-\hat{\mathbf{x}}_{k}^{-}\right)-\mathbf{K}_{k} \boldsymbol{\rho}_{k}\right]\left[\left(\mathbf{x}_{k}-\hat{\mathbf{x}}_{k}^{-}\right)-\mathbf{K}_{k} \boldsymbol{\rho}_{k}\right]^{\top}\right\} \\
& =\mathcal{E}\left[\left(\mathbf{x}_{k}-\hat{\mathbf{x}}_{k}^{-}\right)\left(\mathbf{x}_{k}-\hat{\mathbf{x}}_{k}^{-}\right)^{\top}-\mathbf{K}_{k} \boldsymbol{\rho}_{k}\left(\mathbf{x}_{k}-\hat{\mathbf{x}}_{k}^{-}\right)^{\top}-\left(\mathbf{x}_{k}-\hat{\mathbf{x}}_{k}^{-}\right) \boldsymbol{\rho}_{k}^{\top} \mathbf{K}_{k}^{\top}+\mathbf{K}_{k} \boldsymbol{\rho}_{k} \boldsymbol{\rho}_{k}^{\top} \mathbf{K}_{k}^{\top}\right] \\
& =\mathbf{P}_{k}^{-}-\mathbf{K}_{k}\left(\mathbf{P}_{k \mid k-1}^{x y}\right)^{\top}-\mathbf{P}_{k \mid k-1}^{x y} \mathbf{K}_{k}^{\top}+\mathbf{K}_{k} \mathbf{P}_{k \mid k-1}^{y y} \mathbf{K}_{k}^{\top} .
\end{aligned}
$$

Substituting Eqs. (6) and (8) into Eq. (7) yields

$$
\begin{aligned}
\hat{J}_{k} & =\operatorname{tr}\left[\mathbf{P}_{k}^{-}-\mathbf{K}_{k}\left(\mathbf{P}_{k \mid k-1}^{x y}\right)^{\top}-\mathbf{P}_{k \mid k-1}^{x y} \mathbf{K}_{k}^{\top}+\mathbf{K}_{k} \mathbf{P}_{k \mid k-1}^{y y} \mathbf{K}_{k}^{\top}\right] \\
& +\lambda_{k}\left[\left(\hat{\mathbf{x}}_{k}^{-}\right)^{\top} \hat{\mathbf{x}}_{k}^{-}+2\left(\hat{\mathbf{x}}_{k}^{-}\right)^{\top} \mathbf{K}_{k} \boldsymbol{\rho}_{k}+\boldsymbol{\rho}_{k}^{\top} \mathbf{K}_{k}^{\top} \mathbf{K}_{k} \boldsymbol{\rho}_{k}-\ell^{2}\right] .
\end{aligned}
$$

To find the optimal $\mathbf{K}_{k}$ that minimizes $\hat{J}_{k}$, the derivative of $\hat{J}_{k}$ with respect to $\mathbf{K}_{k}$ is taken and set equal to zero:

$$
\frac{\partial \hat{J}_{k}}{\partial \mathbf{K}_{k}}=-2 \mathbf{P}_{k \mid k-1}^{x y}+2 \mathbf{K}_{k} \mathbf{P}_{k \mid k-1}^{y y}+2 \lambda_{k} \hat{\mathbf{x}}_{k}^{-} \boldsymbol{\rho}_{k}^{\top}+2 \lambda_{k} \mathbf{K}_{k} \boldsymbol{\rho}_{k} \boldsymbol{\rho}_{k}^{\top}=\mathbf{0} .
$$

Solving for $\mathbf{K}_{k}$ yields

$$
\mathbf{K}_{k}=\left(\mathbf{P}_{k \mid k-1}^{x y}-\lambda_{k} \hat{\mathbf{x}}_{k}^{-} \boldsymbol{\rho}_{k}^{\top}\right)\left(\mathbf{P}_{k \mid k-1}^{y y}+\lambda_{k} \mathbf{K}_{k} \boldsymbol{\rho}_{k} \boldsymbol{\rho}_{k}^{\top}\right)^{-1} .
$$

Using the matrix inversion lemma results in

$$
\begin{aligned}
\mathbf{K}_{k} & =\mathbf{P}_{k \mid k-1}^{x y}\left(\mathbf{P}_{k \mid k-1}^{y y}\right)^{-1}-\frac{\lambda_{k} \mathbf{P}_{k \mid k-1}^{x y}\left(\mathbf{P}_{k \mid k-1}^{y y}\right)^{-1} \boldsymbol{\rho}_{k} \boldsymbol{\rho}_{k}^{\top}\left(\mathbf{P}_{k \mid k-1}^{y y}\right)^{-1}}{1+\lambda_{k} \boldsymbol{\rho}_{k}^{\top}\left(\mathbf{P}_{k \mid k-1}^{y y}\right)^{-1} \boldsymbol{\rho}_{k}}-\lambda_{k} \hat{\mathbf{x}}_{k}^{-} \boldsymbol{\rho}_{k}^{\top}\left(\mathbf{P}_{k \mid k-1}^{y y}\right)^{-1} \\
& +\frac{\lambda_{k}^{2} \hat{\mathbf{x}}_{k}^{-} \boldsymbol{\rho}_{k}^{\top}\left(\mathbf{P}_{k \mid k-1}^{y y}\right)^{-1} \boldsymbol{\rho}_{k} \boldsymbol{\rho}_{k}^{\top}\left(\mathbf{P}_{k \mid k-1}^{y y}\right)^{-1}}{1+\lambda_{k} \boldsymbol{\rho}_{k}^{\top}\left(\mathbf{P}_{k \mid k-1}^{y y}\right)^{-1} \boldsymbol{\rho}_{k}} .
\end{aligned}
$$

Substituting Eq. (9) into Eq. (6) results in a quadratic equation in $\lambda_{k}$

$$
a \lambda_{k}^{2}+b \lambda_{k}+c=0
$$


where $a=-\rho_{k}^{2} \ell^{2}, b=-2 \rho_{k} \ell^{2}$,

$$
\begin{aligned}
c & =\boldsymbol{\rho}_{k}^{\top}\left(\mathbf{P}_{k \mid k-1}^{y y}\right)^{-1}\left(\mathbf{P}_{k \mid k-1}^{x y}\right)^{\top} \mathbf{P}_{k \mid k-1}^{x y}\left(\mathbf{P}_{k \mid k-1}^{y y}\right)^{-1} \boldsymbol{\rho}_{k}+2\left(\hat{\mathbf{x}}_{k}^{-}\right)^{\top} \mathbf{P}_{k \mid k-1}^{x y}\left(\mathbf{P}_{k \mid k-1}^{y y}\right)^{-1} \boldsymbol{\rho}_{k}+\left(\hat{\mathbf{x}}_{k}^{-}\right)^{\top} \hat{\mathbf{x}}_{k}^{-}-\ell^{2} \\
& =\left(\hat{\mathbf{x}}_{k}^{-}+\mathbf{P}_{k \mid k-1}^{x y}\left(\mathbf{P}_{k \mid k-1}^{y y}\right)^{-1} \boldsymbol{\rho}_{k}\right)^{\top}\left(\hat{\mathbf{x}}_{k}^{-}+\mathbf{P}_{k \mid k-1}^{x y}\left(\mathbf{P}_{k \mid k-1}^{y y}\right)^{-1} \boldsymbol{\rho}_{k}\right)-\ell^{2},
\end{aligned}
$$

and $\rho_{k}=\boldsymbol{\rho}_{k}^{\top}\left(\mathbf{P}_{k \mid k-1}^{y y}\right)^{-1} \boldsymbol{\rho}_{k}$. This equation has two roots:

$$
\begin{aligned}
\lambda_{k} & =\frac{-b \pm \sqrt{b^{2}-4 a c}}{2 a}=\frac{2 \rho_{k} \ell^{2} \pm \sqrt{4 \rho_{k}^{2} \ell^{4}+4 \rho_{k}^{2} \ell^{2} c}}{-2 \rho_{k}^{2} \ell^{2}}=\frac{1 \pm \sqrt{1+c / \ell^{2}}}{-\rho_{k}} \\
& =-\frac{1}{\rho_{k}} \pm\left[-\frac{\left\|\hat{\mathbf{x}}_{k}^{-}+\mathbf{P}_{k \mid k-1}^{x y}\left(\mathbf{P}_{k \mid k-1}^{y y}\right)^{-1} \boldsymbol{\rho}_{k}\right\|}{\rho_{k} \ell}\right] .
\end{aligned}
$$

To minimize the objective function, the negative root is chosen:

$$
\begin{aligned}
\lambda_{k} & =-\frac{1}{\rho_{k}}-\left[-\frac{\left\|\hat{\mathbf{x}}_{k}^{-}+\mathbf{P}_{k \mid k-1}^{x y}\left(\mathbf{P}_{k \mid k-1}^{y y}\right)^{-1} \boldsymbol{\rho}_{k}\right\|}{\rho_{k} \ell}\right] \\
& =-\frac{1}{\rho_{k}}+\frac{\left\|\hat{\mathbf{x}}_{k}^{-}+\mathbf{P}_{k \mid k-1}^{x y}\left(\mathbf{P}_{k \mid k-1}^{y y}\right)^{-1} \boldsymbol{\rho}_{k}\right\|}{\rho_{k} \ell} .
\end{aligned}
$$

Recognizing that $\tilde{\mathbf{K}}_{k}=\mathbf{P}_{k \mid k-1}^{x y}\left(\mathbf{P}_{k \mid k-1}^{y y}\right)^{-1}$ is the unconstrained Kalman gain matrix, and denoting

$$
\tilde{\mathbf{x}}_{k}=\hat{\mathbf{x}}_{k}^{-}+\tilde{\mathbf{K}}_{k} \boldsymbol{\rho}_{k},
$$

we can express the Lagrange multiplier as

$$
\lambda_{k}=-\frac{1}{\rho_{k}}+\frac{\left\|\hat{\mathbf{x}}_{k}^{-}+\tilde{\mathbf{K}}_{k} \boldsymbol{\rho}_{k}\right\|}{\rho_{k} \ell}=-\frac{1}{\rho_{k}}+\frac{\left\|\tilde{\mathbf{x}}_{k}\right\|}{\rho_{k} \ell} .
$$

Substituting Eq. (11) into Eq. (9) yields the optimal gain matrix

$$
\mathbf{K}_{k}^{*}=\tilde{\mathbf{K}}_{k}+\frac{1}{\rho_{k}}\left(\frac{\ell}{\left\|\tilde{\mathbf{x}}_{k}\right\|}-1\right) \tilde{\mathbf{x}}_{k} \boldsymbol{\rho}_{k}^{\top}\left(\mathbf{P}_{k \mid k-1}^{y y}\right)^{-1} .
$$

Using this gain matrix, the corrected state and covariance matrix are then given by

$$
\begin{aligned}
\hat{\mathbf{x}}_{k} & =\hat{\mathbf{x}}_{k}^{-}+\mathbf{K}_{k}^{*} \boldsymbol{\rho}_{k}, \\
\mathbf{P}_{k} & =\mathbf{P}_{k}^{-}-\mathbf{K}_{k}^{*}\left(\mathbf{P}_{k \mid k-1}^{x y}\right)^{\top}-\mathbf{P}_{k \mid k-1}^{x y}\left(\mathbf{K}_{k}^{*}\right)^{\top}+\mathbf{K}_{k}^{*} \mathbf{P}_{k \mid k-1}^{y y}\left(\mathbf{K}_{k}^{*}\right)^{\top} .
\end{aligned}
$$

Making the substitutions $\mathbf{P}_{k \mid k-1}^{x y}=\mathbf{P}_{k}^{-} \mathbf{H}_{k}^{\top}$ and $\mathbf{P}_{k \mid k-1}^{y y}=\mathbf{H}_{k} \mathbf{P}_{k}^{-} \mathbf{H}_{k}^{\top}+\mathbf{R}_{k}$ arrives at the norm-constrained Kalman filter as presented in Ref. 26. However if the substitutions $\mathbf{P}_{k \mid k-1}^{x y}=\mathbf{U}_{k}$ and $\mathbf{P}_{k \mid k-1}^{y y}=\mathbf{V}_{k}$ are made, where $\mathbf{U}_{k}$ and $\mathbf{V}_{k}$ are defined in Eq. (2) and Eq. (3), then a norm-constrained UKF is obtained.

Now consider the case where only part of the state is constrained with $\hat{\mathbf{x}}_{k}=\left[\begin{array}{ll}\hat{\mathbf{x}}_{1, k}^{\top} & \hat{\mathbf{x}}_{2, k}^{\top}\end{array}\right]^{\top}$, such that $\hat{\mathbf{x}}_{1, k}$ satisfies a norm constraint and $\hat{\mathbf{x}}_{2, k}$ is unconstrained. The Kalman gain and covariance matrices can then be partitioned:

$$
\mathbf{K}_{k}=\left[\begin{array}{l}
\mathbf{K}_{1, k} \\
\mathbf{K}_{2, k}
\end{array}\right], \quad \quad \mathbf{U}_{k}=\left[\begin{array}{c}
\mathbf{U}_{1, k} \\
\mathbf{U}_{2, k}
\end{array}\right], \quad \quad \mathbf{P}_{k}=\left[\begin{array}{ll}
\mathbf{P}_{11, k} & \mathbf{P}_{12, k} \\
\mathbf{P}_{21, k} & \mathbf{P}_{22, k}
\end{array}\right] .
$$

These partitioned matrices can then be substituted into the updated covariance of Eq. (5), arriving at

$$
\mathbf{P}_{k}=\left[\begin{array}{ll}
\mathbf{P}_{11, k}^{-} & \mathbf{P}_{12, k}^{-} \\
\mathbf{P}_{21, k}^{-} & \mathbf{P}_{22, k}^{-}
\end{array}\right]-\left[\begin{array}{c}
\mathbf{K}_{1, k} \\
\mathbf{K}_{2, k}
\end{array}\right]\left[\begin{array}{ll}
\mathbf{U}_{1, k}^{\top} & \mathbf{U}_{2, k}^{\top}
\end{array}\right]-\left[\begin{array}{c}
\mathbf{U}_{1, k} \\
\mathbf{U}_{2, k}
\end{array}\right]\left[\begin{array}{ll}
\mathbf{K}_{1, k}^{\top} & \mathbf{K}_{2, k}^{\top}
\end{array}\right]+\left[\begin{array}{l}
\mathbf{K}_{1, k} \\
\mathbf{K}_{2, k}
\end{array}\right] \mathbf{V}_{k}\left[\begin{array}{ll}
\mathbf{K}_{1, k}^{\top} & \mathbf{K}_{2, k}^{\top}
\end{array}\right] .
$$

The partitions of the updated covariance are then

$$
\mathbf{P}_{11, k}=\mathbf{P}_{11, k}^{-}-\mathbf{K}_{1, k} \mathbf{U}_{1, k}^{\top}-\mathbf{U}_{1, k} \mathbf{K}_{1, k}^{\top}+\mathbf{K}_{1, k} \mathbf{V}_{k} \mathbf{K}_{1, k}^{\top},
$$




$$
\begin{aligned}
& \mathbf{P}_{12, k}=\mathbf{P}_{12, k}^{-}-\mathbf{K}_{1, k} \mathbf{U}_{2, k}^{\top}-\mathbf{U}_{1, k} \mathbf{K}_{2, k}^{\top}+\mathbf{K}_{1, k} \mathbf{V}_{k} \mathbf{K}_{2, k}^{\top}, \\
& \mathbf{P}_{21, k}=\mathbf{P}_{12, k}^{\top}, \\
& \mathbf{P}_{22, k}=\mathbf{P}_{22, k}^{-}-\mathbf{K}_{2, k} \mathbf{U}_{2, k}^{\top}-\mathbf{U}_{2, k} \mathbf{K}_{2, k}^{\top}+\mathbf{K}_{2, k} \mathbf{V}_{k} \mathbf{K}_{2, k}^{\top} .
\end{aligned}
$$

It is apparent that $\mathbf{P}_{11, k}$ and $\mathbf{P}_{22, k}$ are dependent on their respective partitions of the Kalman gain matrix, $\mathbf{K}_{1, k}$ and $\mathbf{K}_{2, k}$. Since the objective function of the minimum covariance filter is dependent on the trace of $\mathbf{P}_{k}$,

$$
J_{k}=\operatorname{trace}\left\{\mathbf{P}_{k}\right\}=\operatorname{trace}\left\{\mathbf{P}_{11, k}\right\}+\operatorname{trace}\left\{\mathbf{P}_{22, k}\right\} .
$$

Furthermore, since $\mathbf{P}_{11, k}$ is only dependent on $\mathbf{K}_{1, k}$ and $\mathbf{P}_{22, k}$ is only dependent on $\mathbf{K}_{2, k}$, then minimizing $J_{k}$ with respect to $\mathbf{K}_{k}$ allows for each partition of $\mathbf{K}_{k}$ to be calculated independently. Consequently $\mathbf{K}_{1, k}$ will be given by Eq. (12) and $\mathbf{K}_{2, k}$ will be given by Eq. (4).

\section{Application to Flat Plate Space-Debris Object Tracking}

\section{A. Dynamics Model}

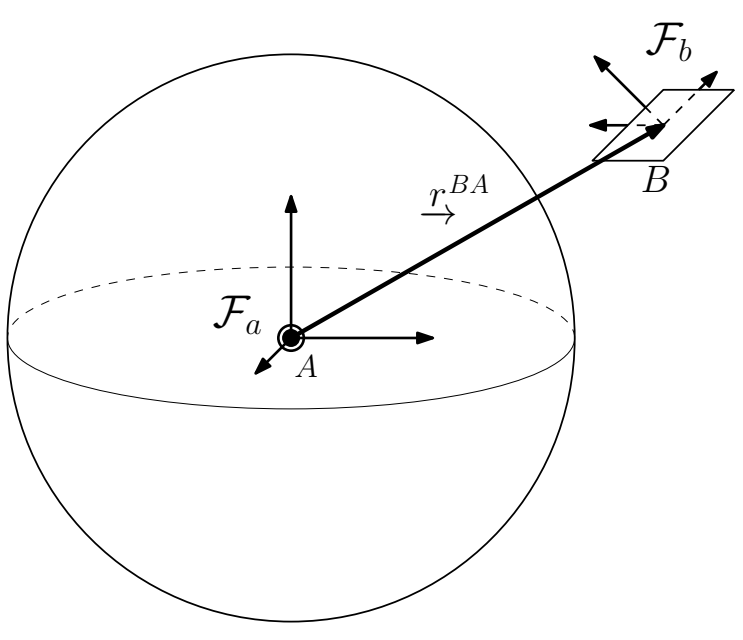

Figure 1. ECI and body-fixed frames.

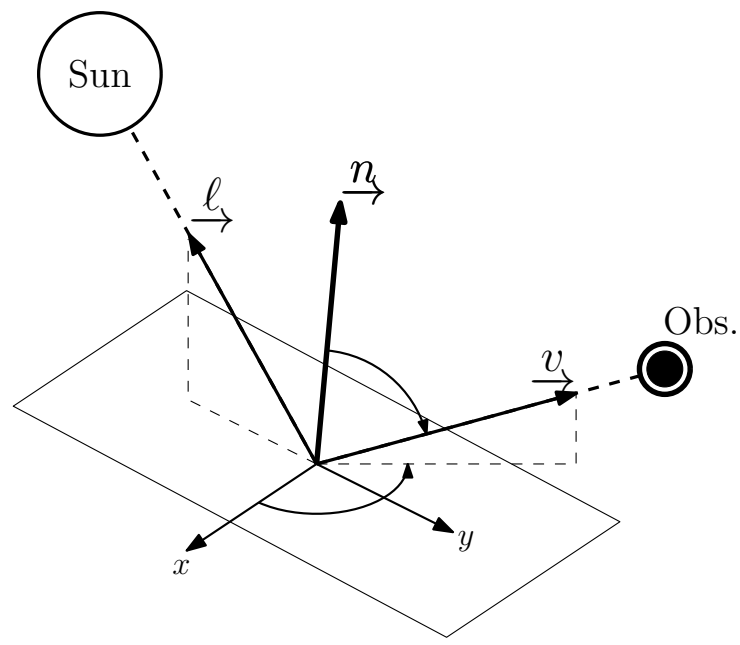

Figure 2. Surface reflection geometry.

It is desired to apply the norm-constrained UKF to estimate the pose of a HAMR space object. In order to accomplish this objective, dynamics and measurement models are needed. To describe the state of a space object in orbit, two frames are defined, the Earth Centered Inertial (ECI) frame $\mathcal{F}_{a}$ and the body-fixed frame $\mathcal{F}_{b}$ as illustrated in Fig. 1 . In the present, only the subproblem of estimating the pose of the debris object without estimating the material properties and mass is considered. Consequently, the object's state is described by

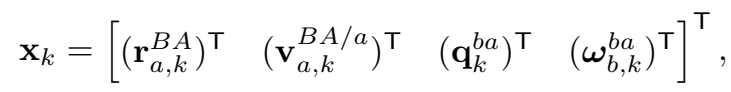

which includes the object's position $\mathbf{r}_{a, k}^{B A}$ relative to the center of the Earth and resolved in $\mathcal{F}_{a}$, the object's velocity $\mathbf{v}_{a, k}^{B A / a}$ relative to the center of the Earth with respect to $\mathcal{F}_{a}$ and resolved in $\mathcal{F}_{a}$, the attitude of the object's body-fixed frame $\mathcal{F}_{b}$ relative to the $\mathcal{F}_{a}$ described using a quaternion $\mathbf{q}_{k}^{b a}=\left[\left(\boldsymbol{\epsilon}_{k}^{b a}\right)^{\top} \eta_{k}^{b a}\right]^{\top}$, and the angular velocity $\boldsymbol{\omega}_{b, k}^{b a}$ of the object's body-fixed frame relative to $\mathcal{F}_{a}$ resolved in $\mathcal{F}_{b}$. For HAMR objects in high-altitude orbits near the GEO belt, the dominant perturbation force is due to the SRP. As a result the translational dynamics are taken to be

$$
\underline{a}_{\rightarrow}^{B A / a}=\stackrel{f}{f}^{g}+\underline{f}^{\mathrm{SRP}}+\stackrel{f}{\rightarrow}^{\mathrm{d}},
$$

where $m$ is the object's mass, $\underline{a}^{B A / a}$ is the acceleration of the object relative to Earth's center with respect to $\mathcal{F}_{a}, f_{\rightarrow}^{g}$ is the force acting on the object due to gravity including $J_{2}$ perturbations (Ref. 29, p. 150, 164), 
$f_{\rightarrow}^{\text {SRP }}$ is the force due to the effect of the SRP, and ${\underset{f}{f}}^{\text {d }}$ is a disturbance force to account for unmodeled

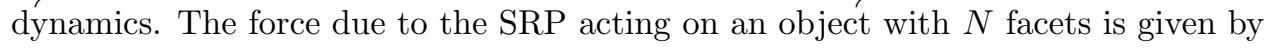

$$
\stackrel{f}{\rightarrow}^{\mathrm{SRP}}=-\sum_{i=1}^{N} \frac{\Phi_{\text {Sun }, \text { tot }} A_{i} \max \left\{0,\left(\underline{\ell} \rightarrow \underline{n_{i}}\right)\right\}}{\left(d / d_{0}\right)^{2} c}\left[2\left(\frac{C_{d, i}}{3}+C_{s, i}\left(\underline{\ell}_{\rightarrow} \cdot \underline{n}_{i}\right)\right) \underline{n}_{i}+\left(1-C_{s, i}\right) \underline{\ell}_{\rightarrow}\right],
$$

where $\Phi_{\text {Sun,tot }}=1367\left[\mathrm{~W} / \mathrm{m}^{2}\right]$ is the total solar flux over all wavelengths at $1[\mathrm{AU}]$ from the Sun, $A_{i}$ is the area of the $i^{\text {th }}$ surface, $\ell$ is the direction of the Sun as illustrated in Fig. 2, $\underline{n}_{i}$ is the normal of the $i^{\text {th }}$ surface, $\left(d / d_{0}\right)$ is the ratio of the distance of the Earth from the Sun to 1 [AU], $c$ is the speed of light, $C_{d, i}$ is the diffuse reflectance of the $i^{\text {th }}$ facet, and $C_{s, i}$ is the specular reflectance at normal incidence of the $i^{\text {th }}$ facet (Ref. 30, p. 582). The direction of the Sun, $\stackrel{\ell}{\rightarrow}$, can be found using the algorithm described in (Ref. 30, p. 279).

The attitude dynamics is governed by Euler's equation

$$
\mathbf{J}_{b} \dot{\boldsymbol{\omega}}_{b}^{b a}+\left(\boldsymbol{\omega}_{b}^{b a}\right)^{\times} \mathbf{J}_{b} \boldsymbol{\omega}_{b}^{b a}=\boldsymbol{\tau}_{b}^{\mathrm{SRP}}+\boldsymbol{\tau}_{b}^{\mathrm{d}},
$$

where $\mathbf{J}_{b}$ is the object's inertia matrix resolved in the body-fixed frame $\mathcal{F}_{b}$, and $(\cdot)^{\times}$is the cross operator (Ref. 31, p. 526). The moment on the space object due to the SRP effects resolved in the object's body frame is

$$
\boldsymbol{\tau}_{b}^{\mathrm{SRP}}=\sum_{i=1}^{N}\left(\mathbf{r}_{b, i}^{C B}\right)^{\times} \mathbf{f}_{b, i}^{\mathrm{SRP}}
$$

where $\mathbf{r}_{b, i}^{C B}$ is the position the center of the $i^{\text {th }}$ surface relative to the body's center of mass, and $\mathbf{f}_{b, i}^{\mathrm{SRP}}$ is the force due to the SRP on the $i^{\text {th }}$ surface. ${ }^{3}$ The term $\tau_{b}^{\text {d }}$ is a disturbance torque to account for unmodeled dynamics. The attitude kinematics is given by

$$
\dot{\mathbf{q}}^{b a}=\boldsymbol{\Xi} \boldsymbol{\omega}_{b}^{b a}, \quad \boldsymbol{\Xi}=\frac{1}{2}\left[\begin{array}{c}
\eta^{b a} \mathbf{I}_{3 \times 3}+\left(\boldsymbol{\epsilon}^{b a}\right)^{\top} \\
-\left(\boldsymbol{\epsilon}^{b a}\right)^{\top}
\end{array}\right],
$$

where the quaternion is partitioned as $\mathbf{q}^{b a}=\left[\left(\boldsymbol{\epsilon}^{b a}\right)^{\boldsymbol{\top}} \eta^{b a}\right]^{\top}$. The quaternion also satisfies the norm-constraint $\left(\mathbf{q}_{k}^{b a}\right)^{\top} \mathbf{q}_{k}^{b a}=1$.

\section{B. Measurement Model}

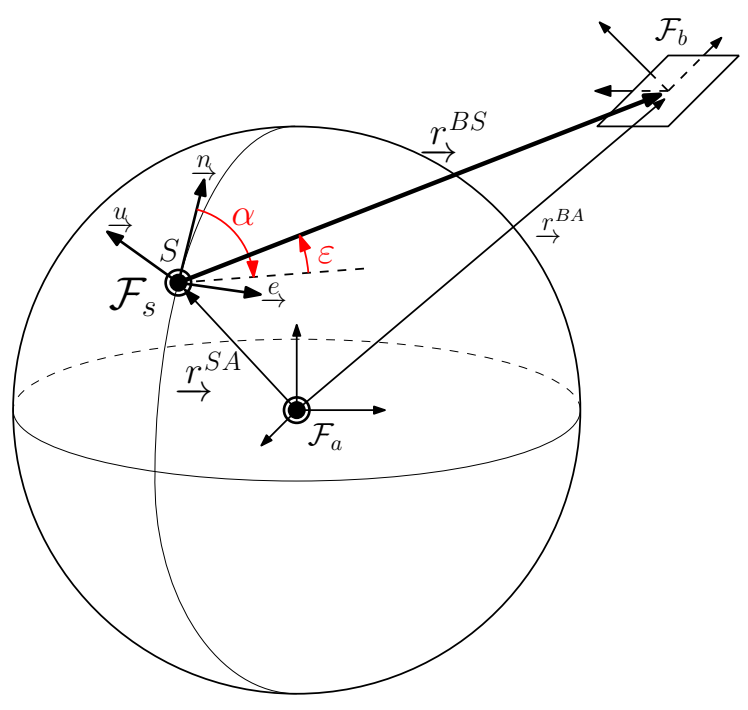

Figure 3. Observation site and up-East-North frame for angles measurements.

Measurements are taken from optical telescopes located at observation sites on Earth. These measurements include azimuth and elevation angles, which provide information on the object's orbit, and a brightness 
magnitude, which gives information on the object's attitude. To determine the angles measurements, the position of the observation site in the ECI frame needs to be determined. The position of the observation site in the ECI frame is given by

$$
\mathbf{r}_{a}^{S A}=\left[\begin{array}{c}
\left\|\underline{r}^{S A}\right\| \cos (\phi) \cos (\lambda) \\
\left\|\underline{r}^{S A}\right\| \sin (\phi) \cos (\lambda) \\
\left\|\underline{r}_{\rightarrow}^{S A}\right\| \sin (\lambda)
\end{array}\right]
$$

where $\left\|r^{S A}\right\|$ is the distance of the observation site from the origin of the ECI frame, $\phi$ is the sidereal time of the observer, and $\lambda$ is the geodetic latitude of the observer. The local up-East-North frame $\mathcal{F}_{s}$ is attached to the observation site as shown in Fig. 3. The position of the space object relative to the observation site resolved in the ECI frame is given by

$$
\mathbf{r}_{a}^{B S}=\mathbf{r}_{a}^{B A}-\mathbf{r}_{a}^{S A}
$$

This position is resolved in frame $\mathcal{F}_{s}$ through the following matrix multiplication

$$
\mathbf{r}_{s}^{B S}=\left[\begin{array}{c}
r_{s, 1}^{B S} \\
r_{s, 2}^{B S} \\
r_{s, 3}^{B S}
\end{array}\right]=\left[\begin{array}{ccc}
\cos (-\lambda) & 0 & -\sin (-\lambda) \\
0 & 1 & 0 \\
\sin (-\lambda) & 0 & \cos (-\lambda)
\end{array}\right]\left[\begin{array}{ccc}
\cos (\phi) & \sin (\phi) & 0 \\
-\sin (\phi) & \cos (\phi) & 0 \\
0 & 0 & 1
\end{array}\right] \mathbf{r}_{a}^{B S}
$$

The azimuth angle $\alpha$ and the elevation angle $\varepsilon$ are then given by

$$
\alpha=\operatorname{atan} 2\left(r_{s, 2}^{B S}, r_{s, 3}^{B S}\right), \quad \varepsilon=\sin ^{-1}\left(\frac{r_{s, 1}^{B S}}{\left\|\mathbf{r}_{s}^{B S}\right\|}\right)
$$

The brightness magnitude measurements are modeled using BRDFs. BRDFs describe the scattering of incident light reflected off a surface in the direction of the viewer $\underline{v}$ as illustrated in Fig. 2. For this application $\underline{v}$ is the direction of the observation site relative to the space object, given by

$$
\underline{v}=-\underline{r}^{B S} /\left\|\underline{r}^{B S}\right\|
$$

BRDFs are typically composed of two components: a specular-like component, $R_{\text {spec }}$, and a diffuse-like component, $R_{\text {diff }}$, such that

$$
R_{\text {total }}=R_{\text {spec }}+R_{\text {diff. }}
$$

For this study, the Ashikhmin-Shirley (AS) BRDF B, $32^{2}$ is used. The specular term of the AS BRDF is given by

$$
R_{\text {spec }}=\frac{\sqrt{\left(n_{u}+1\right)\left(n_{v}+1\right)}}{8 \pi} \frac{(\underline{n} \cdot \underline{h})^{n_{u} \cos ^{2} \beta+n_{v} \sin ^{2} \beta}}{(\underline{h} \cdot \underline{v}) \max [(\underline{n} \cdot \underline{\ell}),(\underline{n} \cdot \underline{v})]} F_{\text {Fresnel }}
$$

where $\stackrel{h}{\rightarrow}$ is the bisector between $\stackrel{\ell}{\rightarrow}$ and $\stackrel{v}{\rightarrow}, n_{u}$ and $n_{v}$ are anisotropic exponential factors, $\beta$ is the angle between $\underline{h}$ and local surface vectors used to define the anisotropic reflection behaviour, and $F_{\text {Fresnel }}$ is the Fresnel reflectance. The AS BRDF approximates $F_{\text {Fresnel }}$ using Schlick's approximation:

$$
F_{\text {Fresnel }}=C_{s}+\left(1-C_{s}\right)(1-(\underline{v} \cdot \underline{h}))^{5} .
$$

The diffuse component is given by

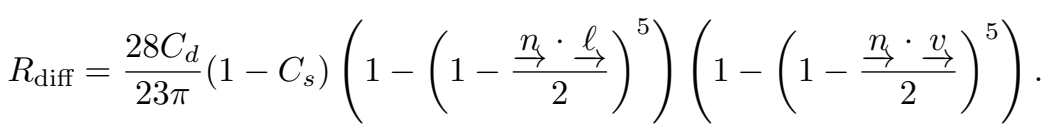

Using this BRDF, the power of the visible light reflected off a surface of the space object toward the observation site is given by

$$
F_{\text {obs }, i}=R_{\text {total }, i} \frac{\Phi_{\text {Sun }, \mathrm{vis}} A_{i} \max \left\{0,\left(\underline{n}_{i} \cdot \underline{\ell}\right)\right\} \max \left\{0,\left(\underline{n}_{\rightarrow} \cdot \underline{v}_{\rightarrow}\right)\right\}}{\left\|\underline{r}_{\rightarrow}^{B S}\right\|^{2}}
$$


where $\Phi_{\text {Sun,vis }}=455\left[\mathrm{~W} / \mathrm{m}^{2}\right]$ is the power per square meter of visible light striking the surface, ${ }^{3} A_{i}$, is the area of the $i^{\text {th }}$ facet, and the maximums of the dot-products or zeros account for instances when the surface is not reflecting Sunlight in the direction of the observer. ${ }^{3}$ The apparent brightness magnitude measured at the observation site is then given by ${ }^{3}$

$$
m_{\mathrm{app}}=-26.7-2.5 \log _{10}\left(\sum_{i=1}^{N} \frac{F_{\mathrm{obs}, i}}{\Phi_{\mathrm{Sun}, \mathrm{vis}}}\right)
$$

where the apparent magnitude of the Sun is -26.7 .

\section{Simulation and Estimation Results}

\section{Measurements from One Observation Site}

With the dynamics and measurement models defined in Sections IV.A and IV.B, the norm-constrained UKF was implemented to estimate the position, velocity, attitude represented by a quaternion, and the angular velocity of a simulated tumbling rigid plate representing debris in a high altitude orbit. In this simulation, the observation site was taken to be at Maui $\mathrm{HI}$ at $20.71^{\circ} \mathrm{N}$ latitude, $156.26^{\circ} \mathrm{W}$ longitude, and 3058.6 $[\mathrm{m}]$ altitude. Measurements were taken every $10[\mathrm{~s}]$ for one hour starting at 04:00.0 Mar 16, 2010 UTC. Measurements were corrupted by zero-mean Gaussian noise with standard deviations 0.1 magnitude for the brightness measurements and 1 arc-second for each of the angles measurements. ${ }^{3,7}$ The plate properties are outlined in Table 1 and the initial state of the plate in the simulation was given by

$$
\mathbf{r}_{a, 0}^{B A}=\left[\begin{array}{c}
-18127.2 \\
31776.3 \\
21128.0
\end{array}\right][\mathrm{km}], \quad \mathbf{v}_{a, 0}^{B A / a}=\left[\begin{array}{c}
-2.6707 \\
-1.5263 \\
0.0073
\end{array}\right][\mathrm{km} / \mathrm{s}], \quad \mathbf{q}_{0}^{b a}=\left[\begin{array}{c}
0.3271 \\
-0.6374 \\
0.5737 \\
-0.3970
\end{array}\right], \quad \boldsymbol{\omega}_{b, 0}^{b a}=\left[\begin{array}{c}
-0.01091 \\
-0.00851 \\
-0.00688
\end{array}\right][\mathrm{rad} / \mathrm{s}] .
$$

For this simulation, the disturbance forces and moments described in Eq. (13) and Eq. (14) were taken to be zero mean Gaussian processes. The disturbance force components had variance $\sigma_{f}^{2}=\left(1 \times 10^{-6}\right)^{2}\left[\mathrm{~N}^{2}\right]$ and the disturbance torque components had variance $\sigma_{\tau}^{2}=\left(1 \times 10^{-8}\right)^{2}\left[(\mathrm{~N} \cdot \mathrm{m})^{2}\right]$. Both the disturbance forces and moments were taken to have characteristic length-scales of $20[\mathrm{~s}]$, and were modeled using the method outlined in Refs. 33 and 34. Using these parameters, the plate's dynamics were simulated in MATLAB using a built-in variable step ordinary differential equation solver. The simulated plate's states were then used to calculate simulated measurements that were subsequently corrupted with zero-mean Gaussian noise. These simulated measurements are shown in Fig. 4.

\begin{tabular}{lcc}
\hline \hline Property & Symbol & Value \\
\hline Length & $a$ & $5[\mathrm{~m}]$ \\
Width & $b$ & $2[\mathrm{~m}]$ \\
Area-to-Mass Ratio & $1 / \rho$ & $26.3\left[\mathrm{~m}^{2} / \mathrm{kg}\right]$ \\
Specular Reflectance at Normal Incidence & $C_{s}$ & 0.60 \\
Diffuse Reflectance & $C_{d}$ & 0.26 \\
AS BRDF Exponential Factor & $n_{u}=n_{v}$ & 10 \\
\hline \hline
\end{tabular}

Table 1. Plate Properties

In addition to these measurements, an initial estimate, $\hat{\mathbf{x}}_{0}$, and initial state-error covariance, $\mathbf{P}_{0}$, is also required for the UKF. For this study, the initial estimate used for the estimation of the state of the plate had a prescribed deviation from the simulated true state. These initial errors are included in Table 2 and the initial variances are given in Table 3 . Finally the $\mathbf{Q}_{k}$ and $\mathbf{R}_{k}$ matrices used to account for process and measurement noise in the UKF were taken to be

$$
\mathbf{Q}_{k}=\left[\begin{array}{ccc}
\mathbf{I}_{3 \times 3} \times\left(1 \times 10^{-6}\right)^{2} & {\left[\mathrm{~N}^{2}\right]} & \mathbf{0} \\
\mathbf{0} & \mathbf{I}_{3 \times 3} \times\left(1 \times 10^{-7}\right)^{2} & {\left[(\mathrm{~N} \cdot \mathrm{m})^{2}\right]}
\end{array}\right],
$$




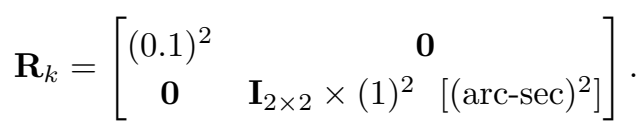

These values constitute Case 1. The norm-constrained UKF of Section III was implemented with the weighting factor $\kappa=3$ and where the states were partitioned such that the quaternion states were norm-constrained and the rest of the states were unconstrained. To improve the norm-constrained UKF's performance, an iterated correction step was implemented as described in Ref. 35. For Case 1, the resulting state estimate errors are shown in Fig. 5 and a visualization of the attitude estimate at different times is shown in Fig. 8. From Fig. 5, it can be seen that the state estimates obtained from the UKF remained within the estimated $3 \sigma$ error bounds calculated from $\mathbf{P}_{k}$. Also, from Fig. 8 it can be seen that the attitude estimate converged to the true attitude using 20 minutes worth of observations and tracked the attitude well for the duration of the observations. It should be noted that although the position error remained bounded, the filter performance was coarse and did not significantly improve over the duration of the observations.

Now consider Case 2, where the stringent initial error and initial variance on the angular velocity were relaxed with $\delta \boldsymbol{\omega}_{b}^{b a}=\mathbf{1}_{3 \times 1} \times 100[\mathrm{deg} / \mathrm{h}]$ and $\sigma_{\omega}^{2}=(200)^{2}\left[(\mathrm{deg} / \mathrm{h})^{2}\right]$. The UKF failed to converge to the true state as illustrated in Fig. 6 and as visualized for the attitude states in Fig. 9. This fragility can be in part ascribed to the lack of information available to the UKF through the light curve measurements. Essentially a single datum point in the form of a brightness magnitude measurement was being used to infer an attitude with three degrees of freedom. Even though the system's dynamics can be used to weave these measurements together to gain more information in the form of light curves, this information is insufficient for the case of a plate, since only one surface is available to reflect the Sunlight at a given time. This contrasts previous work that involved three dimensional structures, since such structures had more facets from which information could be revealed in the measured light curves.

\section{Measurements from Multiple Observation Sites}

If data were available from multiple observation sites, then the state estimate of the plate's motions for a less confident initial angular velocity estimate could be recovered for Case 2. This is illustrated in Fig. 7 where in addition to measurements from Maui HI, synchronized measurements were also taken from Sorroco NM and Las Campanas Chile. The location of these additional observation sites are included in Table 4. From Fig. 7 and Fig. 10, it is apparent that the UKF estimates remained within the estimated $3 \sigma$ bounds again and converged to the true state more quickly and with smaller covariances after one hour of observations. This added robustness further motivates the need for establishing a central framework through which observations from multiple telescope in varied locations can be combined to provide more data on space-debris objects. ${ }^{15}$

\section{Conclusions}

In this study, an unscented Kalman filter that enforces a state equality norm constraint was presented. This was accomplished by modifying the minimum variance estimation problem to be a constrained optimization problem with a Lagrange multiplier as outlined by Zanetti et al. ${ }^{26}$ This norm-constrained unscented Kalman filter was then applied to a space-debris tracking problem with the objective of estimating the pose of a flat plate in a high-altitude orbit using light curve and angles measurements in simulation. It was found that by fusing synchronized measurements from multiple Earth-based observation sites, the ability of the norm-constrained UKF to converge to the true state of the plate's pose was improved whereas taking observations from a single observation site would lead to divergent state estimates. This serves as further motivation for establishing a more diverse Space Surveillance Network with a central measurement database as proposed by programs such as DARPA's OrbitOutlook project. 


\begin{tabular}{|c|c|c|}
\hline Property & Symbol & Value \\
\hline Initial position error & $\delta \mathbf{r}_{a}^{B A}$ & $\mathbf{1}_{3 \times 1} \times 1[\mathrm{~km}]$ \\
\hline Initial velocity error & $\delta \mathbf{v}_{a}^{B A / a}$ & $\mathbf{1}_{3 \times 1} \times 0.001[\mathrm{~km} / \mathrm{s}]$ \\
\hline Initial attitude error & $\delta \mathbf{q}^{b a}$ & {$\left[\begin{array}{ll}\left(\mathbf{1}_{1 \times 3} \times \sin \left(40^{\circ} / 2\right)\right) & \cos \left(40^{\circ} / 2\right)\end{array}\right]$} \\
\hline Initial angular velocity error & $\delta \boldsymbol{\omega}_{b}^{b a}$ & $\mathbf{1}_{3 \times 1} \times 50.0[\mathrm{deg} / \mathrm{h}]$ \\
\hline
\end{tabular}

Table 2. Initial errors in the state estimate.

\begin{tabular}{lcc}
\hline \hline Property & Symbol & Value \\
\hline Initial position variances & $\sigma_{r}^{2}$ & $1^{2}\left[\mathrm{~km}^{2}\right]$ \\
Initial velocity variances & $\sigma_{v}^{2}$ & $(0.001)^{2}\left[(\mathrm{~km} / \mathrm{s})^{2}\right]$ \\
Initial attitude variances & $\sigma_{\theta}^{2}$ & $(0.5)^{2}$ \\
Initial angular velocity variances & $\sigma_{\omega}^{2}$ & $(70.0)^{2}\left[(\mathrm{deg} / \mathrm{h})^{2}\right]$ \\
\hline \hline
\end{tabular}

Table 3. Initial variances for state estimation with the norm-constrained UKF.

\begin{tabular}{lccc}
\hline \hline Location & Latitude & Longitude & Altitude \\
\hline Maui HI & $20.71^{\circ} \mathrm{N}$ & $156.26^{\circ} \mathrm{W}$ & $3058.6[\mathrm{~m}]$ \\
Sorroco NM & $33.82^{\circ} \mathrm{N}$ & $106.66^{\circ} \mathrm{W}$ & $1510.2[\mathrm{~m}]$ \\
Las Campanas Chile & $29.01^{\circ} \mathrm{S}$ & $70.69^{\circ} \mathrm{W}$ & $2515.8[\mathrm{~m}]$ \\
\hline \hline
\end{tabular}

Table 4. Observation site locations.
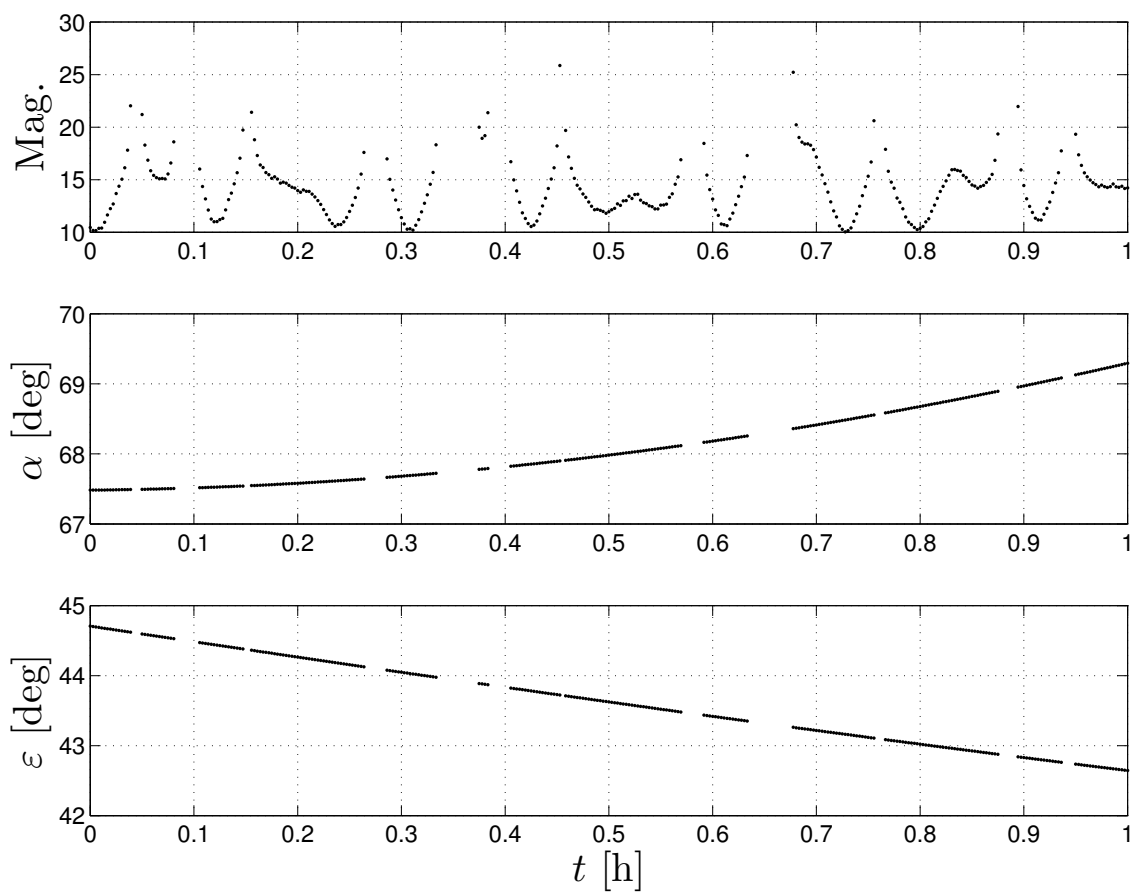

Figure 4. Simulated observations of the space-debris plate from the Maui HI observation site. 

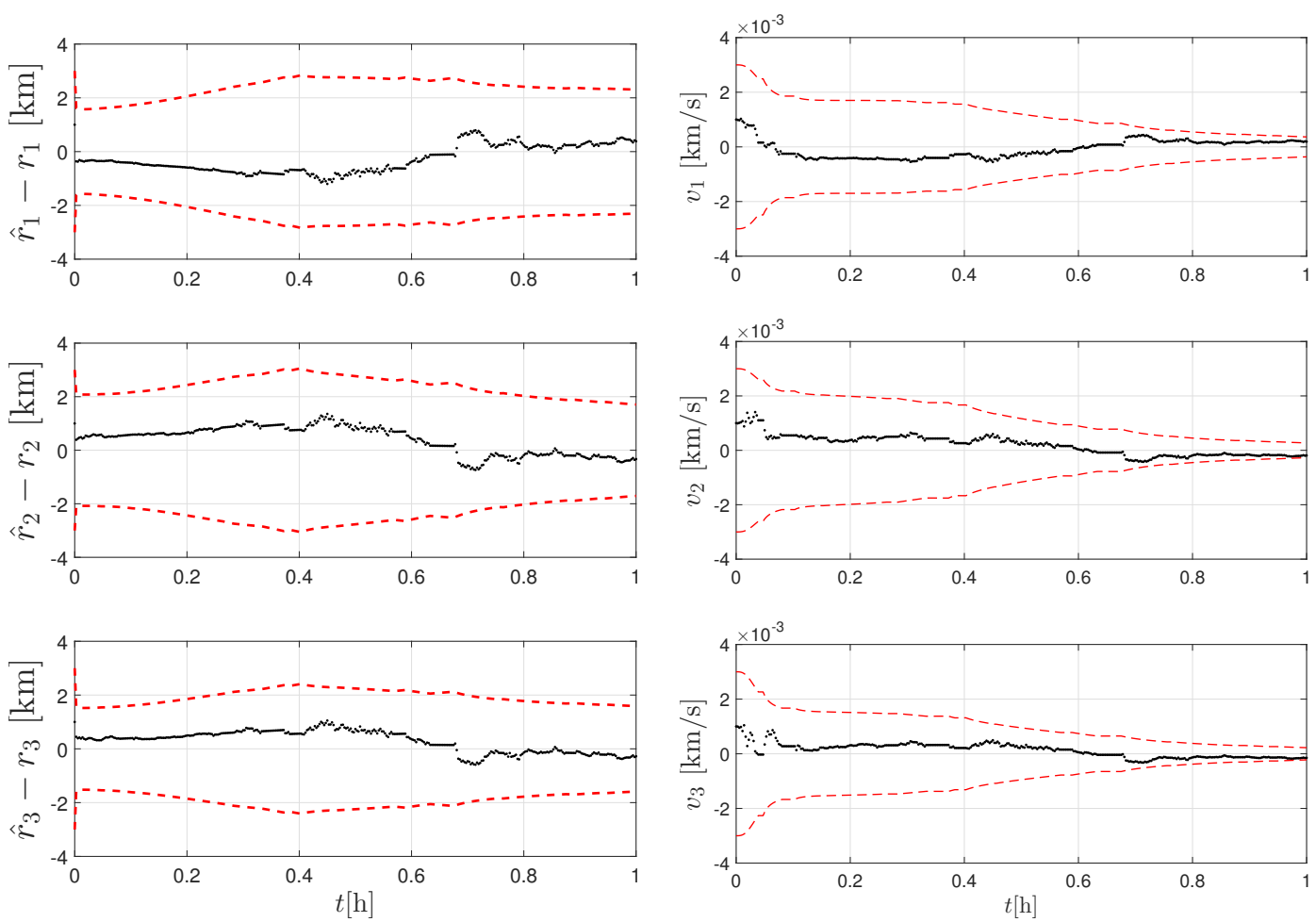

(a) Position Error

(b) Velocity Error
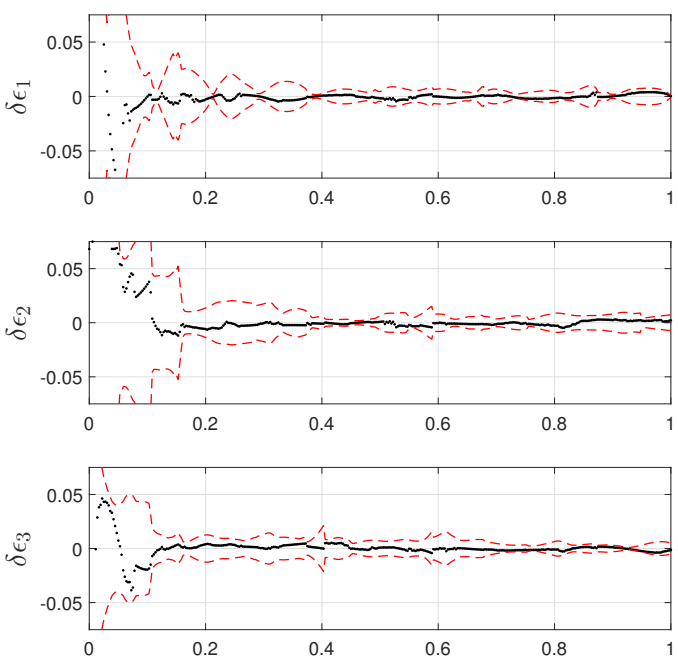

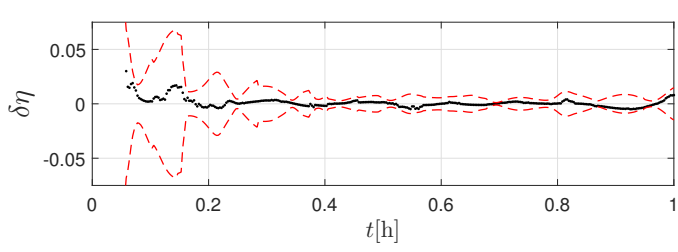

(c) Attitude Error
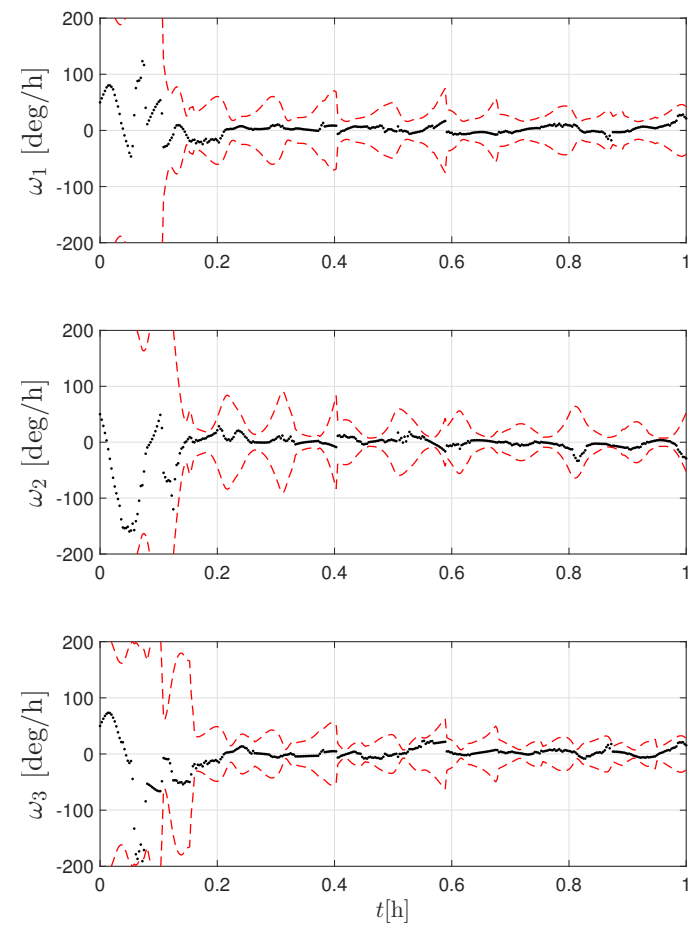

(d) Angular Velocity Error

Figure 5. (Case 1) Estimation errors for tracking the plate from a single observation site. $3 \sigma$ bounds are the red dashed lines. It can be seen that the state estimation errors remain within the estimated $3 \sigma$ bounds. 

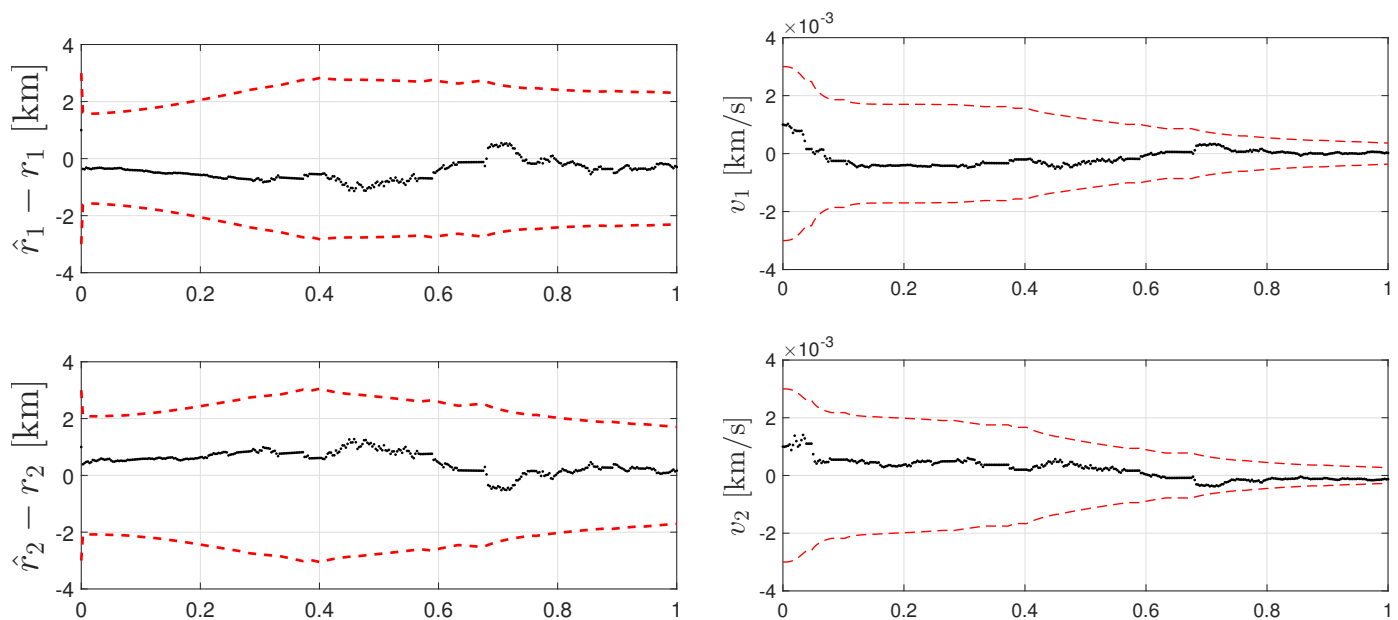

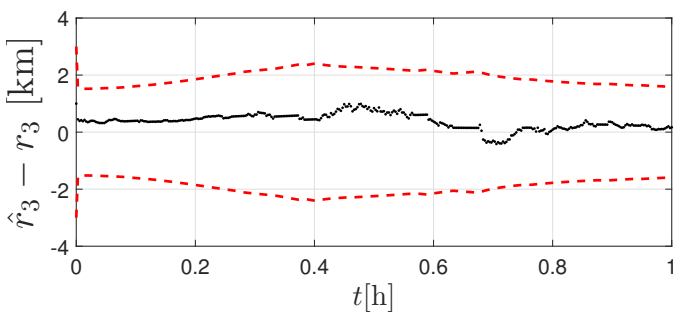

(a) Position Error
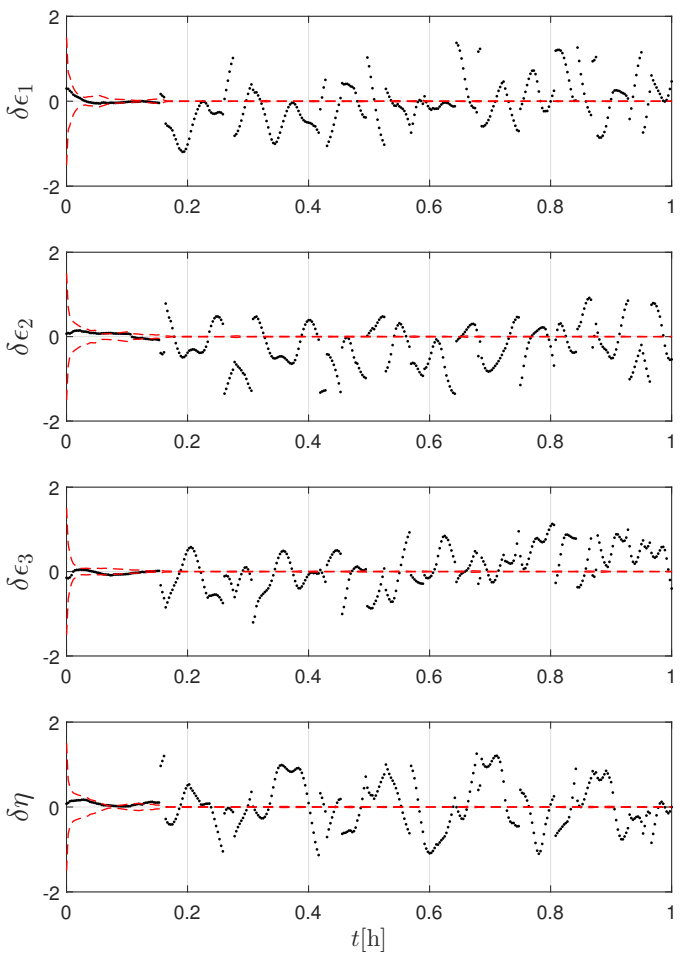

(c) Attitude Error

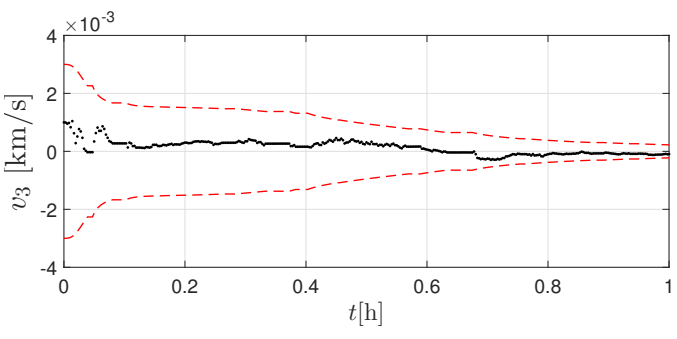

(b) Velocity Error
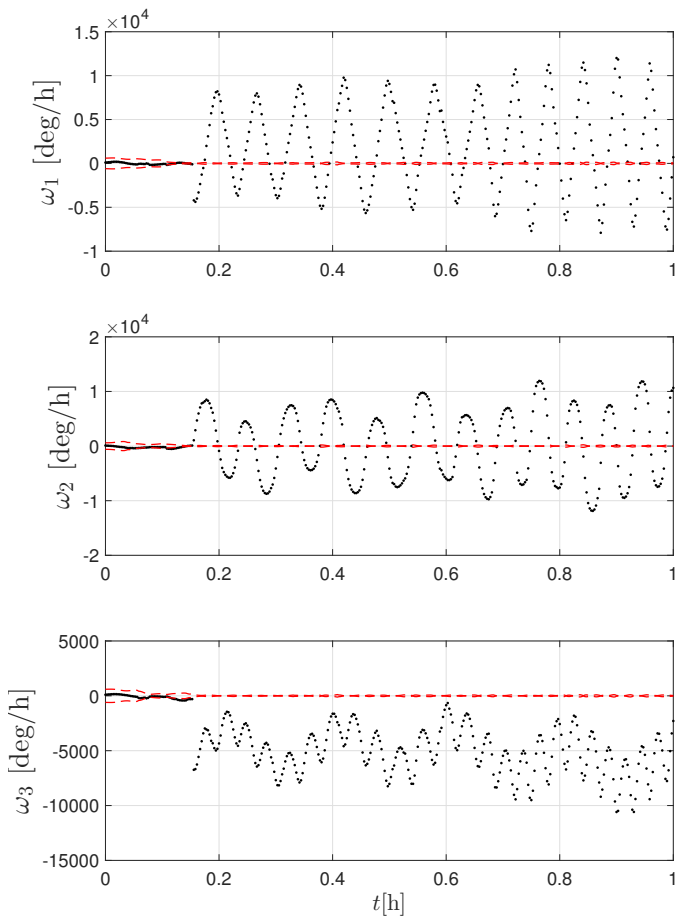

(d) Angular Velocity Error

Figure 6. (Case 2) Estimation error for tracking the plate from a single observation site. It can be seen that the attitude estimates become divergent for a less confident initial guess of the angular velocity. 

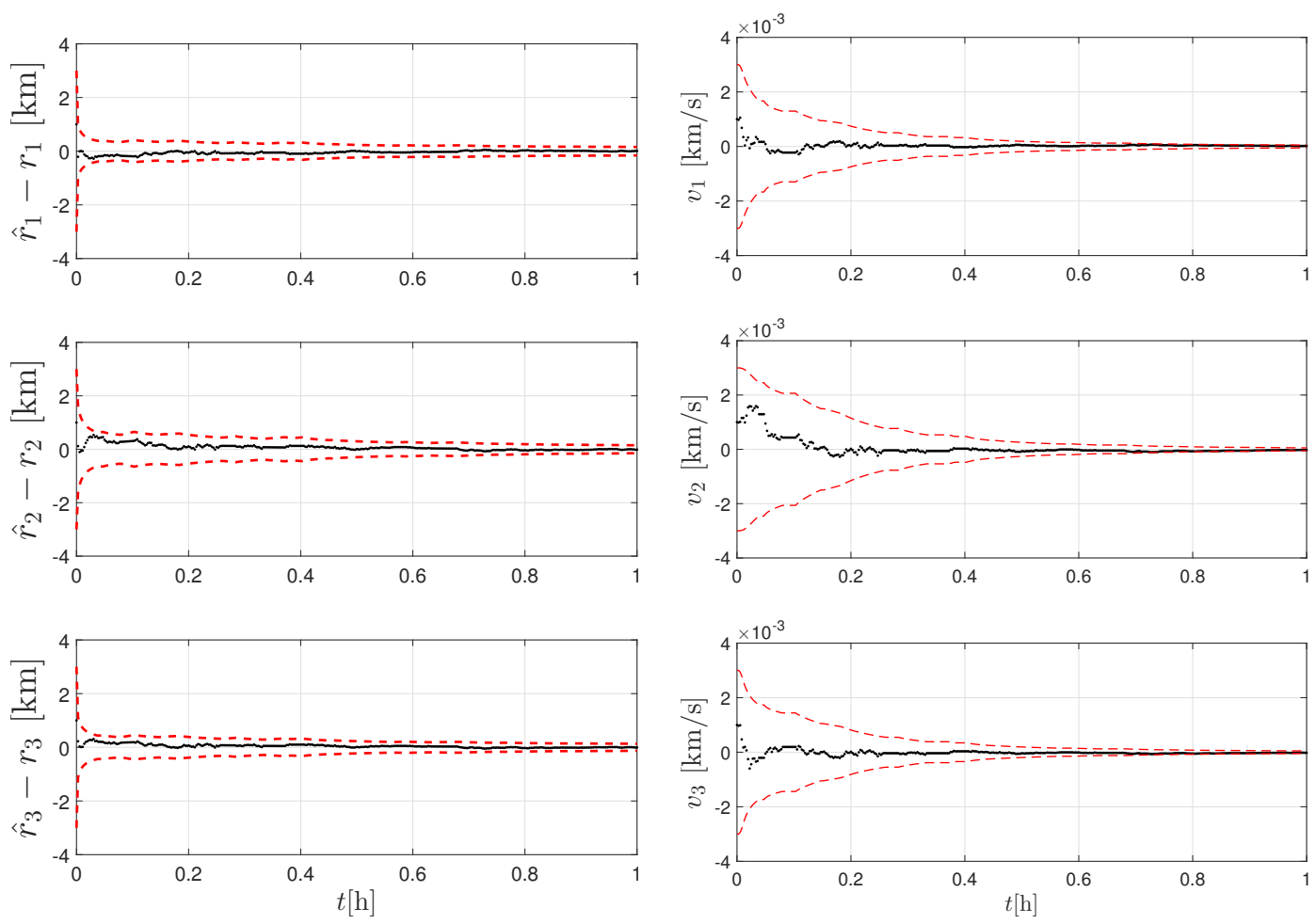

(a) Position Error

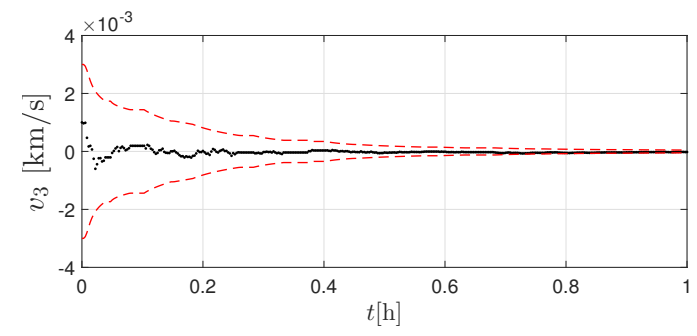

(b) Velocity Error
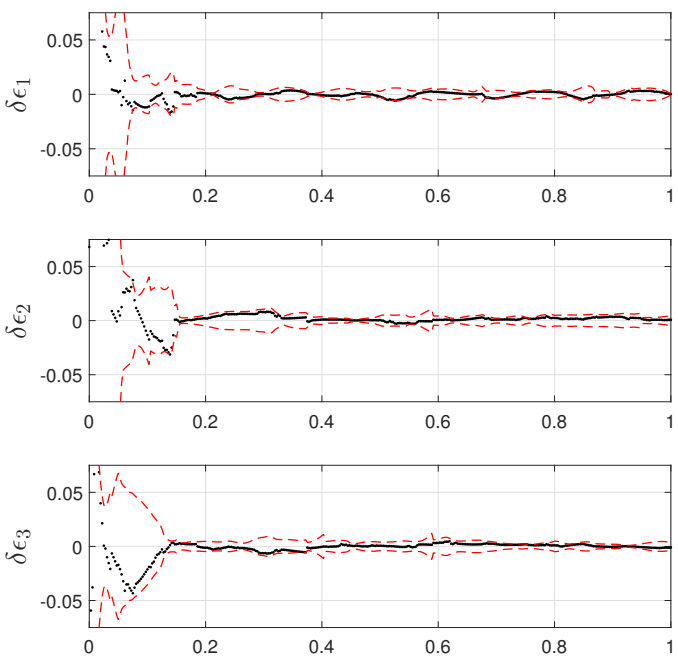

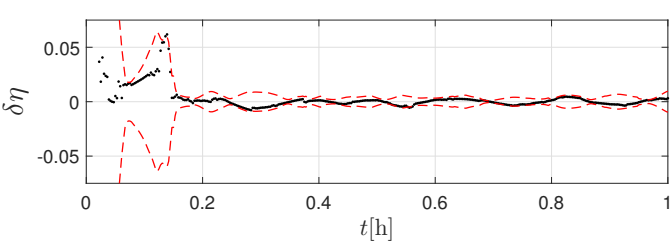

(c) Attitude Error
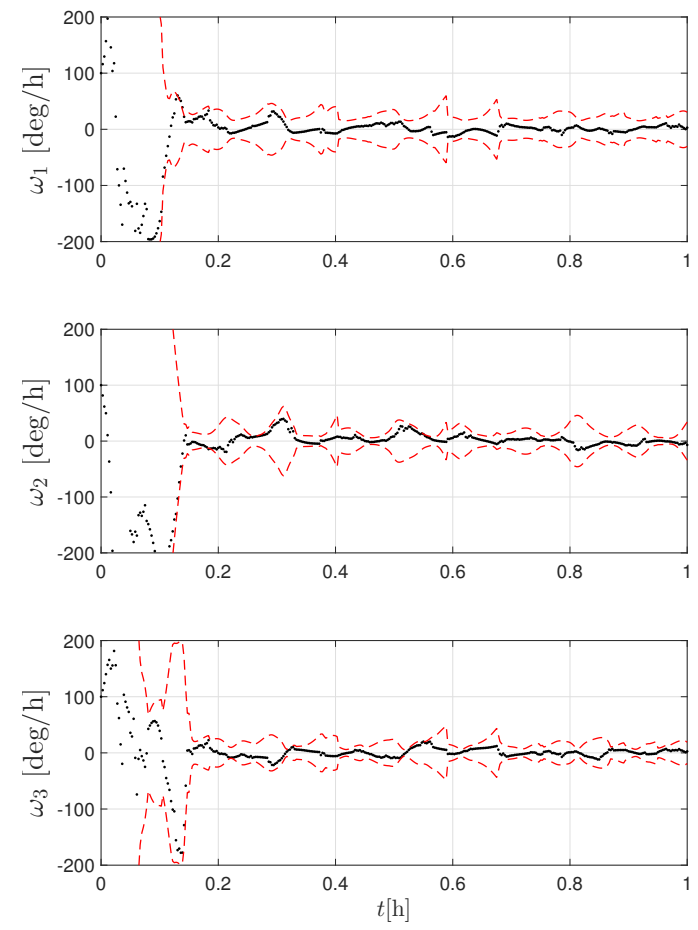

(d) Angular Velocity Error

Figure 7. (Case 2) Estimation error for tracking the plate from three observation sites. It can be seen that the position estimate is noticeably improved in comparison to estimates from a single site. The attitude estimates are also convergent. 


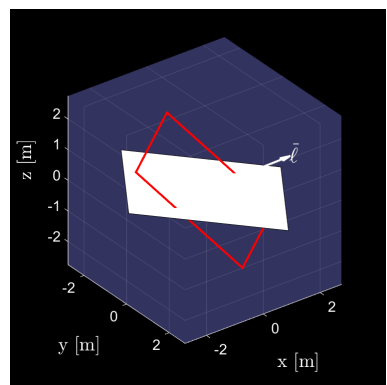

(a) $t=0 \min$

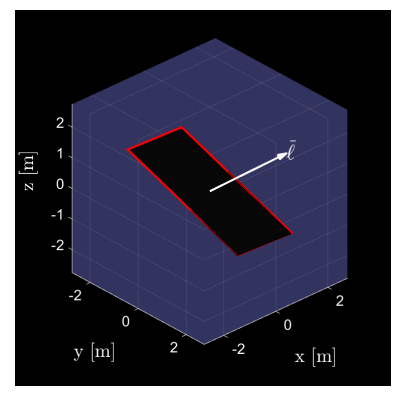

(b) $t=20 \mathrm{~min}$

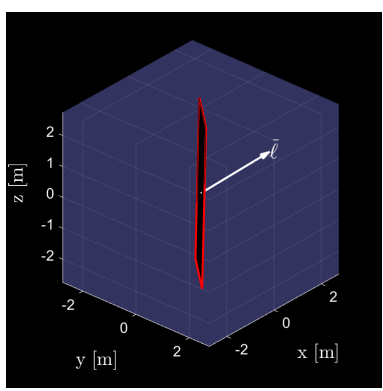

(c) $t=40 \mathrm{~min}$

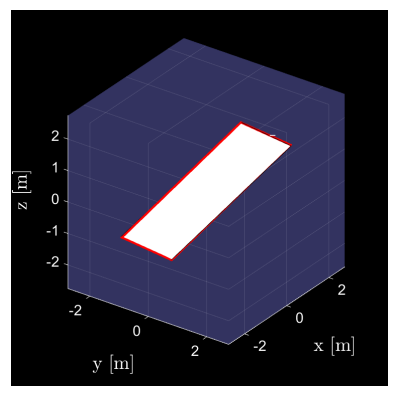

(d) $t=60 \mathrm{~min}$

Figure 8. (Case 1) Attitude estimate visualization for a single observation site. The red outline corresponds to the norm-constrained UKF state estimate of the quaternion representation of the attitude. It can be seen that by the $20 \mathrm{~min}$. time step, the attitude estimate has converged to the true state.

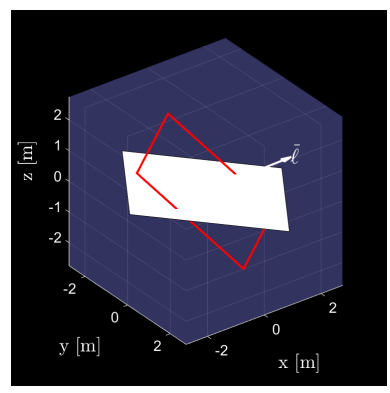

(a) $t=0 \min$

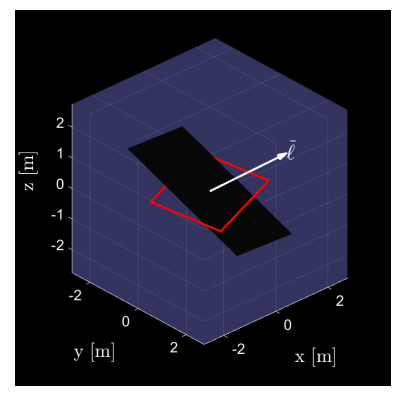

(b) $t=20 \mathrm{~min}$

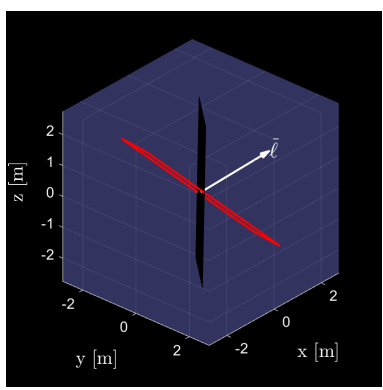

(c) $t=40 \mathrm{~min}$

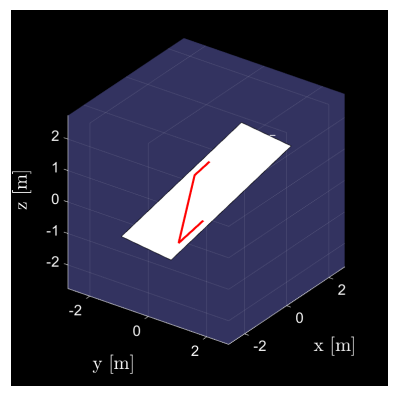

(d) $t=60 \mathrm{~min}$

Figure 9. (Case 2) Attitude estimate visualization for a single observation site. The red outline corresponds to the norm-constrained UKF state estimate of the quaternion representation of the attitude. It can be seen that the attitude estimate does not converge to the true estimate.

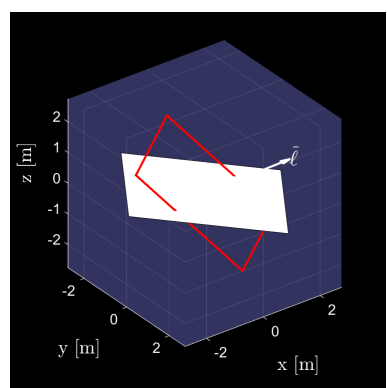

(a) $t=0 \mathrm{~min}$

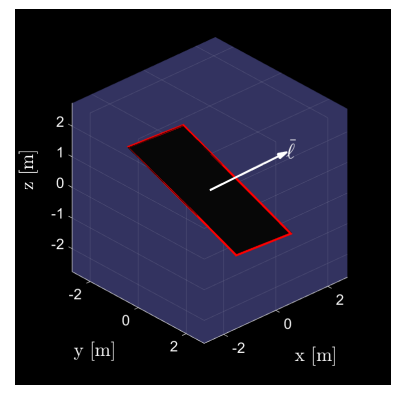

(b) $t=20 \mathrm{~min}$

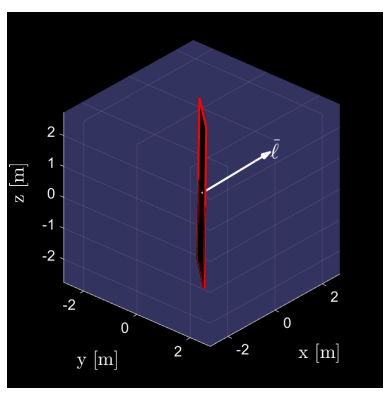

(c) $t=40 \mathrm{~min}$

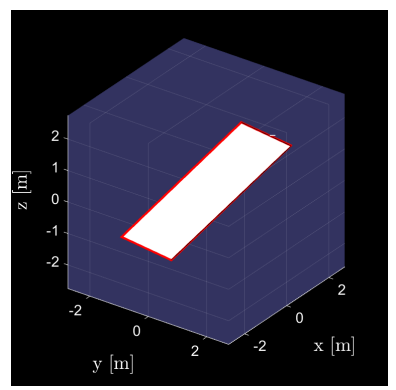

(d) $t=60 \mathrm{~min}$

Figure 10. (Case 2) Attitude estimate visualization for measurements from three observation sites. The red outline corresponds to the norm-constrained UKF state estimate of the quaternion representation of the attitude. It can be seen that the attitude estimate does converge to the true estimate. 


\section{References}

${ }^{1}$ Committee for the Assessment of the U.S. Air Force's Astrodynamic Standards, Aeronautics and Space Engineering Board, Division on Engineering and Physical Sciences, and National Research Council, Continuing Kepler's Quest: Assessing Air Force Space Command's Astrodynamics Standards., The National Academies Press, Washington, DC, 2012.

${ }^{2}$ Schildknecht, T., "Optical Surveys for Space Debris," The Astronomy and Astrophysics Review, Vol. 14, No. 1, January 2007, pp. 41-111. doi:10.1007/s00159-006-0003-9.

${ }^{3}$ Linares, R., Jah, M. K., Crassidis, J. L., and Nebelecky, C. K., "Space Object Shape Characterization and Tracking Using Light Curve and Angles Data," Journal of Guidance, Control, and Dynamics, Vol. 37, No. 1, January-February 2014, pp. 13-25. doi:10.2514/1.62986.

${ }^{4}$ Wetterer, C. and Jah, M. K., "Attitude Estimation from Light Curves," Journal of Guidance, Control, and Dynamics, Vol. 32, No. 5, September-October 2009, pp. 1648-1651. doi:10.2514/1.44254.

${ }^{5}$ Linares, R., Jah, M. K., Crassidis, J. L., Leve, F. A., and Kelecy, T. M., "Astrometric and Photometric Data Fusion for Inactive Space Object Mass and Area Estimation," Acta Astronautica, Vol. 99, 2014, pp. 1-15. doi:10.1016/j.actaastro.2013.10.018.

${ }^{6}$ Holzinger, M. J., Alfriend, K. T., Wetterer, C. J., Luu, K. K., Sabol, C., and Hamada, K., "Photometric Attitude Estimation for Agile Space Objects with Shape Uncertainty," Journal of Guidance, Control, and Dynamics, Vol. 37, 2014, pp. 921-932. doi:10.2514/1.58002.

${ }^{7}$ Hall, D. T., Africano, J. L., Lambert, J. V., and Kervin, P. W., "Time-Resolved I-Band Photometry of Calibration Spheres and NaK Droplets," Journal of Spacecraft and Rockets, Vol. 44, No. 4, July-August 2007, pp. 910-919. doi:10.2514/1.27464.

${ }^{8}$ Santoni, F., Cordelli, E., and Piergentili, F., "Determination of Disposed-Upper-Stage Attitude motion by Ground-Based Optical Observations," Journal of Spacecraft and Rockets, Vol. 50, 2013, pp. 701-708. doi:10.2514/1.A32372.

${ }^{9}$ Wetterer, C. J., Linares, R., Crassidis, J. L., Kelecy, T. M., Ziebart, M. K., Jah, M. K., and Cefola, P. J., "Refining Space Object Radiation Pressure Modeling with Bidirectional Reflectance Distribution Functions," Journal of Guidance, Control, and Dynamics, Vol. 37, No. 1, January-February 2014, pp. 185-196. doi:10.2514/1.60577.

${ }^{10}$ Früh, C. and Schildknecht, T., "Attitude Motion of Space Debris Objects Under Influence of Solar Radiation Pressure and Gravity," 633 International Astronautical Congress, International Astronautical Federation, Paris France, 2012, pp. $2350-2355$.

${ }^{11}$ Früh, C., Kelecy, T. M., and Jah, M. K., "Coupled Orbit-Attitude Dynamics of High Area-to-Mass ratio (HAMR) Objects: Influence of Solar Radiation Pressure, Earth's Shadow and Visibility in Light Curves," Celestial Mechanics and Dynamical Astronomy, Vol. 117, No. 4, December 2013, pp. 385-404. doi:10.1007/s10569-013-9516-5.

${ }^{12}$ Früh, C. and Jah, M. K., "Coupled Orbit-Attitude Motion of High Area-to-Mass Ratio (HAMR) Objects Including Efficient Self-Shadowing," Acta Astronautica, Vol. 95, 2014, pp. 227-241. doi:10.1016/j.actaastro.2013.11.017.

${ }^{13}$ Früh, C. and Jah, M. K., "Attitude and Orbit Propagation of High Area-to-Mass Ratio (HAMR) Objects Using a SemiCoupled Approach," The Journal of the Astronautical Sciences, Vol. 60, No. 1, March 2013, pp. 32-50. doi:10.1007/s40295014-0013-1.

${ }^{14}$ Henderson, L. S. and Subbarao, K., "Sensitivity Analysis of the Lightcurve Measurement Model for Use in Attitude and Shape Estimation of Resident Space Objects," Advances of the Astronautical Sciences, Vol. 148, Univelt, Inc., 2013, pp. $3565-3582$.

${ }^{15}$ Blake, T., Sanchez, M., and Bolden, M., "OrbitOutlook: Data-centric Competition Based Space Domain Awareness," $30^{\text {th }}$ Space Symposium Technical Track, Space Foundation, Colorado Springs, CO, May 2014.

${ }^{16}$ Piergentili., F., Ceruti, A., Rizzitelli, R., Cardona, T., Battagliere, M. L., and Santoni, F., "Space Debris Measurement Using Joint Mid-Latitude and Equatorial Optical Observations," IEEE Transactions on Aerospace and Electronic Systems, Vol. 50, No. 1, January 2014. doi:10.1109/TAES.2013.120272.

${ }^{17}$ Markley, F. L., "Attitude Error Representations for Kalman Filtering," Journal of Guidance, Control, and Dynamics, Vol. 26, No. 2, March-April 2003, pp. 311-317. doi:10.2514/2.5048.

${ }^{18}$ Crassidis, J. L., "Unscented Filtering for Spacecraft Attitude Estimation," Journal of Guidance, Control, and Dynamics, Vol. 26, No. 4, July-August 2003, pp. 536-542. doi:10.2514/2.5102.

${ }^{19}$ Alouani, A. T. and Blair, W. D., "Use of a Kinematic Constraint in Tracking Constant Speed, Maneuvering Targets," IEEE Transactions on Automatic Control, Vol. 38, No. 7, July 1993, pp. 1107-1111. doi:10.1109/9.231465.

${ }^{20}$ Richards, P. W., "Constrained Kalman Filtering Using Pseudo-Measurements," Proceedings of the IEE Colloquium on Algorithms for Target Tracking, London, UK, 1995, pp. 75-79. doi:10.1049/ic:19950676.

${ }^{21}$ Gupta, N., "Kalman Filtering in the Presence of State Space Equality Constraints," Proc. of the 26 ${ }^{\text {th }}$ Chinese Control Conference, Zhangjiajie, Hunan, China, July 26-31, 2007, pp. 107-113. doi:10.1109/CHICC.2006.4347158.

${ }^{22}$ Simon, D., "Kalman Filtering with State Constraints: A Survey of Linear and Nonlinear Algorithms," IET Control Theory and Applications, Vol. 4, No. 8, 2010, pp. 1303-1318. doi:10.1049/iet-cta.2009.0032.

${ }^{23}$ Simon, D. and Chia, T. L., "Kalman Filtering with State Equality Constraints," IEEE Transactions on Aerospace and Electronic Systems, Vol. 38, No. 1, January 2002, pp. 128-136. doi:10.1109/7.993234.

${ }^{24}$ Yang, C. and Blasch, E., "Kalman Filtering with Nonlinear State Constraints," IEEE Transactions on Aerospace and Electronic Systems, Vol. 45, No. 1, January 2009, pp. 70-84. doi:10.1109/TAES.2009.4805264.

${ }^{25}$ Julier, S. J. and LaViola, J. J., "On Kalman Filtering with Nonlinear Equality Constraints," IEEE Transactions on Signal Processing, Vol. 55, No. 6, June 2007, pp. 2774-2784. doi:10.1109/TSP.2007.893949.

${ }^{26}$ Zanetti, R., Majji, M., Bishop, R. H., and Mortari, D., "Norm-Constrained Kalman Filtering," Journal of Guidance, Control, and Dynamics, Vol. 32, No. 5, September-October 2009, pp. 1458-1465. doi:10.2514/1.43119.

${ }^{27}$ Simon, D., Optimal State Estimation: Kalman, $H_{\infty}$, and Nonlinear Approaches, John Wiley \& Sons, Inc., Hoboken, NJ, 2006.

${ }^{28}$ Särkkä, S., Bayesian Filtering and Smoothing, Cambridge University Press, New York, NY, 2013. 
${ }^{29}$ de Ruiter, A. H. J., Damaren, C. J., and Forbes, J. R., Spacecraft Dynamics and Control: An Introduction, John Wiley \& Sons, Ltd., West Sussex, United Kingdom, 2013.

${ }^{30}$ Vallado, D. A. and McClain, W. D., Fundamentals of Astrodynamics and Applications, Microcosm Press and Springer, Hawthorne, CA, fourth edition ed., 2013.

${ }^{31}$ Hughes, P. C., Spacecraft Attitude Dynamics, Dover Publications, Inc., Mineola, NY, 2004.

${ }^{32}$ Ashikhmin, M. and Shirley, P., "An Anisotropic Phong BRDF Model," Journal of Graphics Tools, Vol. 5, No. 2, 2000, pp. 25-32. doi:10.1080/10867651.2000.10487522.

${ }^{33}$ Zlotnik, D. E. and Forbes, J. R., "Dynamic Modelling, Estimation, and Control for Precision Pointing of an Atmospheric Balloon Platform," Machines, and Mechatronics Symposium 2013, Canadian Committee for the Theory of Machines and Mechanisms, May 2013.

${ }^{34}$ Rasmussen, C. E. and Williams, C. K. I., Approach Processes for Machine Learning, MIT Press, Cambridge MA, 2006.

${ }^{35}$ Sibley, G., Sukhatme, G., and Matthies, L., "The Iterated Sigma Point Kalman Filter with Applications to Long Range Stereo," Robotics: Science and Systems, Vol. 8, 2006, pp. 235-244. 